\title{
Local times for systems of non-linear stochastic heat equations
}

\author{
Brahim Boufoussi • Yassine Nachit
}

Received: date / Accepted: date

\begin{abstract}
We consider $u(t, x)=\left(u_{1}(t, x), \cdots, u_{d}(t, x)\right)$ the solution to a system of non-linear stochastic heat equations in spatial dimension one driven by a $d$-dimensional space-time white noise. We prove that, when $d \leq 3$, the local time $L(\xi, t)$ of $\{u(t, x), t \in[0, T]\}$ exists and $L(\bullet, t)$ belongs a.s. to the Sobolev space $H^{\alpha}\left(\mathbb{R}^{d}\right)$ for $\alpha<\frac{4-d}{2}$, and when $d \geq 4$, the local time does not exist. We also show joint continuity and establish Hölder conditions for the local time of $\{u(t, x), t \in[0, T]\}$. These results are then used to investigate the irregularity of the coordinate functions of $\{u(t, x), t \in[0, T]\}$. Comparing to similar results obtained for the linear stochastic heat equation (i.e., the solution is Gaussian), we believe that our results are sharp. Finally, we get a sharp estimate for the partial derivatives of the joint density of $\left(u\left(t_{1}, x\right)-u\left(t_{0}, x\right), \cdots, u\left(t_{n}, x\right)-u\left(t_{n-1}, x\right)\right)$, which is a new result and of independent interest.
\end{abstract}

Keywords Local time - Stochastic heat equation · Fourier transform • Malliavin calculus $\cdot$ Space-time white noise

Mathematics Subject Classification (2010) 60H15 $60 \mathrm{~J} 55 \cdot 60 \mathrm{H} 07$

Brahim Boufoussi

Department of Mathematics, Faculty of Sciences Semlalia, Cadi Ayyad University, 2390 Marrakesh, Morocco

E-mail: boufoussi@uca.ac.ma

Yassine Nachit

Department of Mathematics, Faculty of Sciences Semlalia, Cadi Ayyad University, 2390

Marrakesh, Morocco

E-mail: yassine.nachit.fssm@gmail.com 


\section{Introduction and main results}

We consider the following system of non-linear stochastic heat equations

$$
\frac{\partial u_{k}}{\partial t}(t, x)=\frac{\partial^{2} u_{k}}{\partial x^{2}}(t, x)+b_{k}(u(t, x))+\sum_{l=1}^{d} \sigma_{k, l}(u(t, x)) \dot{W}^{l}(t, x),
$$

with Neumann boundary conditions

$$
u_{k}(0, x)=0, \quad \frac{\partial u_{k}(t, 0)}{\partial x}=\frac{\partial u_{k}(t, 1)}{\partial x}=0,
$$

for $1 \leq k \leq d, t \in[0, T], x \in[0,1]$, where $u:=\left(u_{1}, \cdots, u_{d}\right)$. Here, $\dot{W}=$ $\left(\dot{W}^{1}, \cdots, \dot{W}^{d}\right)$ is a vector of $d$-independent space-time white noises on $[0, T] \times$ $[0,1]$. We put $b=\left(b_{k}\right)_{1 \leq k \leq d}$ and $\sigma=\left(\sigma_{k, l}\right)_{1 \leq k, l \leq d}$. Following Walsh [21], we will give, in Section 2.3, a rigorous formulation of the formal equation (1.1). Let us state the following hypotheses on the coefficients $\sigma_{k, l}$ and $b_{k}$ of the system of non-linear stochastic heat equations (1.1):

A1 For all $1 \leq k, l \leq d$, the functions $\sigma_{k, l}$ and $b_{k}$ are bounded and infinitely differentiable such that the partial derivatives of all orders are bounded.

A2 The matrix $\sigma$ is uniformly elliptic i.e., there exists $\rho>0$ such that for all $x \in \mathbb{R}^{d}$ and $z \in \mathbb{R}^{d}$ with $\|z\|=1$, we have $\|\sigma(x) z\|^{2} \geq \rho^{2}$ (where $\|\cdot\|$ is the Euclidean norm on $\mathbb{R}^{d}$ ).

The objective of this paper is to investigate existence and regularity of the local time of $\{u(t, x) ; t \in[0, T]\}$ the solution to Eq. (1.1). The challenge to study local times of $\{u(t, x) ; t \in[0, T]\}$ is twofold: on one hand, $\{u(t, x) ; t \in$ $[0, T]\}$ is neither a Gaussian process nor a stable process in general, on the other hand, the coordinate processes $u_{1}, \cdots, u_{d}$ are not independent. As far as we know, no one has studied the local time of an $\mathbb{R}^{d}$-valued process $X$ with coordinate processes $X_{1}, \cdots, X_{d}$ which are not independent, even in the Gaussian case. The only local times results that we are aware of for nonGaussian processes are 15,13. By conditional Malliavin calculus approach, Lou and Ouyang have established in 15 an upper bound of Gaussian type for the partial derivatives of the n-point joint density of the solution to a stochastic differential equation driven by fractional Brownian motion. Their result is similar to Theorem 1.3(b) below. Furthermore, they have used that result as an alternative to the classical local nondeterminism condition (LND for short), which is often used to investigate local times of Gaussian random fields - for more details on the LND condition one can see 8. Due to this, the authors in 15 have proved the existence and regularity of the local times of stochastic differential equations driven by fractional Brownian motions. Moreover, in 13 , Kerchev et al. have investigated the existence and regularity of the local time of the Rosenblatt process via Berman's method. Their proof is based on a spectral analysis of arbitrary linear combinations of integral operators, which derive from the representation of the Rosenblatt process as an element in the second chaos. The approach adopted here is quite different from the two previous works. Indeed, we mainly use two new tools: we introduce some techniques in 
the Malliavin calculus for adapted stochastic processes (see Section 3) and also a condition involving a local estimation of the characteristic function of the increments of a given process, which we call $\alpha$-local nondeterminism ( $\alpha$-LND for short), see the definition in section 2.1. The role of the $\alpha$-LND condition in our investigation is the same as that of the well-known local nondeterminism condition used in the Gaussian framework. As part of our arguments, by using those techniques of the Malliavin calculus, we show that the systems of nonlinear stochastic heat equations satisfy the $\frac{1}{4}$-LND condition. With this in mind and through Berman's method, we conclude the existence and joint continuity of the local time. Roughly speaking, we believe that the approach presented here can be used to investigate the local times of adapted stochastic processes that are smooth in the Malliavin sense. The main result of our studying is summarized as follows:

Theorem 1.1 Let $u(t, x)$ be the solution to Eq. (1.1), and $x \in(0,1)$ be fixed.

(i) Almost surely, when $d \leq 3$, the local time $L(\xi, t)$ of the process $\{u(t, x), t \in$ $[0, T]\}$ exists for any fixed $t$, moreover, $L(\bullet, t)$ belongs to the Sobolev space $H^{\alpha}\left(\mathbb{R}^{d}\right)$ of index $\alpha<\frac{4-d}{2}$; and when $d \geq 4$, the local time does not exist in $L^{2}\left(\mathbb{P} \otimes \lambda_{d}\right)$ for any $t$, where $\lambda_{d}$ is the Lebesgue measure on $\mathbb{R}^{d}$.

(ii) Assume $d \leq 3$, the local time of the process $\{u(t, x), t \in[0, T]\}$ has a version, denoted by $L(\xi, t)$, which is a.s. jointly continuous in $(\xi, t)$, and which is $\gamma$-Hölder continuous in $t$, uniformly in $\xi$, for all $\gamma<1-\frac{d}{4}$ : there exist two random variables $\eta$ and $\delta$ which are almost surely finite and positive such that

$$
\sup _{\xi \in \mathbb{R}^{d}}|L(\xi, t)-L(\xi, s)| \leq \eta|t-s|^{\gamma}
$$

for all $s, t \in[0, T]$ such that $|t-s|<\delta$.

As a consequence, one can get a result on the behavior of the coordinate functions of the solution to Eq. (1.1).

Corollary 1.2 Let $u(t, x)$ be the solution to Eq. (1.1). Assume $d \leq 3$. Then for each $x \in(0,1)$, almost surely, all coordinate functions of $\{u(t, x), t \in[0, T]\}$ are nowhere Hölder continuous of order greater than $\frac{1}{4}$.

When we were investigating local times, we got the following theorem which is interesting in its own right.

Let $x \in(0,1)$ be fixed, and let $\pi_{n}=\left(t_{1}, \cdots, t_{n}\right)$ with $0=t_{0}<t_{1}<$ $\cdots<t_{n} \leq T$. We denote by $p_{\pi_{n}, x}(\xi)$, where $\xi=\left(\xi_{j, l}, 1 \leq j \leq n, 1 \leq\right.$ $l \leq d) \in \mathbb{R}^{n \times d}$, the density of the $\mathbb{R}^{n \times d}$-valued random vector $\left(u\left(t_{1}, x\right)-\right.$ $\left.u\left(t_{0}, x\right), \cdots, u\left(t_{n}, x\right)-u\left(t_{n-1}, x\right)\right)$, where $u\left(t_{i}, x\right)-u\left(t_{i-1}, x\right)=\left(u_{1}\left(t_{i}, x\right)-\right.$ $\left.u_{1}\left(t_{i-1}, x\right), \cdots, u_{d}\left(t_{i}, x\right)-u_{d}\left(t_{i-1}, x\right)\right)$, for $i=1, \cdots, n$. Put also $p_{s, t, x}(y)$ the density of the $\mathbb{R}^{d}$-valued random vector $\left(u_{1}(t, x)-u_{1}(s, x), \cdots, u_{d}(t, x)-\right.$ $\left.u_{d}(s, x)\right)$. Set $\|\cdot\|$ for the Euclidean norm on $\mathbb{R}^{d}$. For all $\xi=\left(\xi_{j, l}, 1 \leq j \leq\right.$ $n, 1 \leq l \leq d) \in \mathbb{R}^{n \times d}$ and $m=\left(m_{j, l}, 1 \leq j \leq n, 1 \leq l \leq d\right)$ where $m_{j, l}$, for 
$j=1, \cdots, n$ and $l=1, \cdots, d$, are nonnegative integers, we introduce

$$
\partial_{\xi}^{m}=\prod_{j=1}^{n} \prod_{l=1}^{d}\left(\frac{\partial}{\partial \xi_{j, l}}\right)^{m_{j, l}} .
$$

Theorem 1.3 Assume A1 and A2. Then we get the following:

(a) There exists a constant $c>0$ such that for any $x \in(0,1), 0 \leq s<t \leq T$, and $y \in \mathbb{R}^{d}$,

$$
p_{s, t, x}(y) \geq \frac{c}{(t-s)^{d / 4}} \exp \left(-\frac{\|y\|^{2}}{c(t-s)^{1 / 2}}\right) .
$$

(b) Let $n$ be a positive integer and $m_{i, k}$, for $i=1, \cdots, n$ and $k=1, \cdots, d$, be nonnegative integers. Then, there exists a positive constant $c$ (may depend on $n$ and $\left.m_{i, k}\right)$ such that for all $x \in(0,1), \pi_{n}=\left(t_{1}, \cdots, t_{n}\right)$ with $0=t_{0}<t_{1}<\cdots<t_{n} \leq T$, and $\xi=\left(\xi_{j, l}, 1 \leq j \leq n, 1 \leq l \leq d\right) \in \mathbb{R}^{n \times d}$,

$$
\left|\partial_{\xi}^{m} p_{\pi_{n}, x}(\xi)\right| \leq c \prod_{i=1}^{n} \frac{1}{\left(t_{i}-t_{i-1}\right)^{\left(d+\sum_{k=1}^{d} m_{i, k}\right) / 4}} \exp \left(-\frac{\left\|\xi_{i}\right\|^{2}}{c\left(t_{i}-t_{i-1}\right)^{1 / 2}}\right),
$$

where $\xi_{i}=\left(\xi_{i, 1}, \cdots, \xi_{i, d}\right)$ and $m=\left(m_{i, k}, 1 \leq i \leq n, 1 \leq k \leq d\right)$.

In [9, using Malliavin calculus techniques, Dalang et al. have established a Gaussian-type lower bound for the one-point density of the solution $u(t, x)$ and a Gaussian-type upper bound for the two-point density of $(u(s, y), u(t, x))$, in order to get upper and lower bounds for the hitting probabilities of the process $\left\{u(t, x) ; t \in \mathbb{R}^{+}, x \in[0,1]\right\}$. Therefore, we see that Theorem 1.3 generalizes in some sense [9, Theorem 1.1].

Now, recall that a subset $A$ of $\mathbb{R}^{d}$ is polar for an $\mathbb{R}^{d}$-valued stochastic process $\left(X_{t}\right)_{t \in[0, T]}$ if $\mathbb{P}\left[X_{t} \in A\right.$ for some $\left.t\right]=0$. From [9] we know that singletons are not polar for $\{u(t, x), t \in[0, T]\}$ when $d \leq 3$, and are polar for $d \geq 5$. The critical case of dimension $d=4$ is open. We think that there is a deep connection between the polarity and the existence of the local time. Indeed, it is easy to prove that if almost all singletons are polar, then the local time does not exist. As a consequence the local time does not exist for $\{u(t, x), t \in[0, T]\}$ when $d \geq 5$, but in section 5.2, we will give another proof of that, and we will even show that the local time does not exist in the critical dimension $d=4$.

Finally, let us briefly explain that the $\alpha$-LND property (see Definition 2.4) can be a consequence of the integration by parts formula (see Proposition 3.4) as follows:

$$
\mathbb{E}\left[\partial_{y}^{k} e^{i \sum_{j=1}^{n}\left\langle\xi_{j}, u\left(t_{j}, x\right)-u\left(t_{j-1}, x\right)\right\rangle}\right]=\mathbb{E}\left[e^{i \sum_{j=1}^{n}\left\langle\xi_{j}, u\left(t_{j}, x\right)-u\left(t_{j-1}, x\right)\right\rangle} H_{\pi_{n}}^{\beta}(Z, 1)\right],
$$

where $\langle\cdot, \cdot\rangle$ is the Euclidean inner product on $\mathbb{R}^{d}, k=\left(k_{j, l}, 1 \leq j \leq n, 1 \leq l \leq\right.$ $d), \xi=\left(\xi_{j, l}, 1 \leq j \leq n, 1 \leq l \leq d\right)$, and $Z=\left(u\left(t_{1}, x\right)-u\left(t_{0}, x\right), \cdots, u\left(t_{n}, x\right)-\right.$ 
$\left.u\left(t_{n-1}, x\right)\right)$. Our observation is that by the above equality we have,

$$
\prod_{h=1}^{n} \prod_{l=1}^{d}\left|\xi_{h, l}\right|^{k_{h, l}}\left|\mathbb{E}\left[e^{i \sum_{j=1}^{n}\left\langle\xi_{j}, u\left(t_{j}, x\right)-u\left(t_{j-1}, x\right)\right\rangle}\right]\right| \leq \mathbb{E}\left[\left|H_{\pi_{n}}^{\beta}(Z, 1)\right|\right]
$$

One of the main technical efforts in this paper is to estimate $\mathbb{E}\left[\left|H_{\pi_{n}}^{\beta}(Z, 1)\right|\right]$.

The rest of the paper is arranged as follows. In the second section, we give some preliminary results on local times, classical Malliavin calculus, and the stochastic heat equation. The third section is devoted to introducing some new tools in the Malliavin calculus that we will use in the fourth and fifth section in order to prove respectively Theorem 1.3 and Theorem 1.1 .

Finally, we mention that constants in our proofs may change from line to line.

\section{Preliminaries}

\subsection{The local times}

This section is devoted to briefly give some aspects of the theory of local times. For more information on the subject, we refer to the classical paper of Geman and Horowitz 12 .

Let $\left(\theta_{t}\right)_{t \in[0, T]}$ be a Borel function with values in $\mathbb{R}^{d}$. For any Borel set $B \subseteq[0, T]$, the occupation measure of $\theta$ on $B$ is given by the following measure on $\mathbb{R}^{d}$ :

$$
\nu_{B}(\bullet)=\lambda\left\{t \in B ; \theta_{t} \in \bullet\right\},
$$

where $\lambda$ is the Lebesgue measure. When $\nu_{B}$ is absolutely continuous with respect to $\lambda_{d}$ (the Lebesgue measure on $\mathbb{R}^{d}$ ), we say that the local time of $\theta$ on $B$ exists and it is defined, $L(\bullet, B)$, as the Radon-Nikodym derivative of $\nu_{B}$ with respect to $\lambda_{d}$, i.e., for almost every $x$,

$$
L(x, B)=\frac{d \nu_{B}}{d \lambda_{d}}(x) .
$$

In the above, we call $x$ the space variable and $B$ the time variable. We write $L(x, t)$ (resp. $L(x))$ instead of $L(x,[0, t])(\operatorname{resp.} L(x,[0, T]))$.

The local time satisfies the following occupation formula: for any Borel set $B \subseteq[0, T]$, and for every measurable bounded function $f: \mathbb{R}^{d} \rightarrow \mathbb{R}$,

$$
\int_{B} f\left(\theta_{s}\right) d s=\int_{\mathbb{R}^{d}} f(x) L(x, B) d x .
$$

The deterministic function $\theta$ can be chosen to be the sample path of an $\mathbb{R}^{d}$-valued separable stochastic process $X=\left(X_{t}\right)_{t \in[0, T]}$ with $X_{0}=0$ a.s. In this regard, we say that the process $X$ has a local time (resp. square integrable local time) if for almost all $\omega$, the trajectory $t \mapsto X_{t}(\omega)$ has a local time (resp. square integrable local time). 
We investigate the local time via Berman's approach. The idea is to derive properties of $L(\bullet, B)$ from the integrability properties of the Fourier transform of the sample paths of $X$.

Let us state the following hypotheses on the integrability of the characteristic function of $X$ :

B1

$$
\int_{\mathbb{R}^{d}} \int_{0}^{T} \int_{0}^{T} \mathbb{E}\left[e^{i\left\langle u, X_{t}-X_{s}\right\rangle}\right] d t d s d u<\infty
$$

B2 For every even integer $m \geq 2$,

$$
\int_{\left(\mathbb{R}^{d}\right)^{m}} \int_{[0, T]^{m}}\left|\mathbb{E}\left[\exp \left(i \sum_{j=1}^{m}\left\langle u_{j}, X_{t_{j}}\right\rangle\right)\right]\right| \prod_{j=1}^{m} d t_{j} \prod_{j=1}^{m} d u_{j}<\infty .
$$

Recall the following crucial result in [4] (see also [5, Lemma 3.1]):

Theorem 2.1 Assume B1. Then the process $X$ has a square integrable local time. Moreover, we have almost surely, for all Borel set $B \subseteq[0, T]$, and for almost every $x$,

$$
L(x, B)=\frac{1}{(2 \pi)^{d}} \int_{\mathbb{R}^{d}} e^{-i\langle u, x\rangle} \int_{B} e^{i\left\langle u, X_{t}\right\rangle} d t d u .
$$

In (2.1), $L(x, B)$ is not a stochastic process. Following Berman [5] we construct a version of the local time, which is a stochastic process.

The below theorem is given in Berman [5, Theorem 4.1] for $d=1$ and $m=2$, so we will omit the proof.

Theorem 2.2 Assume B1 and B2. Put for all integer $N \geq 1$,

$$
L_{N}(x, t)=\frac{1}{(2 \pi)^{d}} \int_{[-N, N]^{d}} e^{-i\langle u, x\rangle} \int_{0}^{t} e^{i\left\langle u, X_{s}\right\rangle} d s d u
$$

Then there exists a stochastic process $\tilde{L}(x, t)$ separable in the $x$-variable, such that for each even integer $m \geq 2$,

$$
\lim _{N \rightarrow \infty} \sup _{(x, t) \in \mathbb{R}^{d} \times[0, T]} \mathbb{E}\left[\left|L_{N}(x, t)-\tilde{L}(x, t)\right|^{m}\right]=0 .
$$

Theorem 2.3 (Theorem 4.3 in [5]) Let $\tilde{L}(x, t)$ be given by (2.2). If the stochastic process $\left\{\tilde{L}(x, t), x \in \mathbb{R}^{d}\right\}$ is almost surely continuous, then it is a continuous (in the $x$-variable) version of the local time on $[0, t]$.

In order to overcome the problem caused by the dependence of the negligible sets on $x, t$, and $\omega$, we will look for a jointly continuous version of the 
local time. For this end, we have by (2.2), for all $x, y \in \mathbb{R}^{d}, t, h \in[0, T]$ such that $t+h \in[0, T]$, and even integer $m \geq 2$,

$$
\begin{aligned}
& \mathbb{E}[\tilde{L}(x+y, t+h)-\tilde{L}(x, t+h)-\tilde{L}(x+y, t)+\tilde{L}(x, t)]^{m}=\frac{1}{(2 \pi)^{m d}} \\
& \times \int_{\left(\mathbb{R}^{d}\right)^{m}} \int_{[t, t+h]^{m}} \prod_{j=1}^{m}\left(e^{-i\left\langle u_{j}, x+y\right\rangle}-e^{-i\left\langle u_{j}, x\right\rangle}\right) \mathbb{E}\left[e^{i \sum_{j=1}^{m}\left\langle u_{j}, X_{t_{j}}\right\rangle}\right] \prod_{j=1}^{m} d t_{j} \prod_{j=1}^{m} d u_{j} \\
& =\frac{1}{(2 \pi)^{m d}} \int_{\left(\mathbb{R}^{d}\right)^{m}} \int_{[t, t+h]^{m}} \prod_{j=1}^{m}\left(e^{-i\left\langle v_{j}-v_{j+1}, x+y\right\rangle}-e^{-i\left\langle v_{j}-v_{j+1}, x\right\rangle}\right) \\
& \times \mathbb{E}\left[e^{i \sum_{j=1}^{m}\left\langle v_{j}, X_{t_{j}}-X_{t_{j-1}}\right\rangle}\right] \prod_{j=1}^{m} d t_{j} \prod_{j=1}^{m} d v_{j},
\end{aligned}
$$

and

$$
\begin{aligned}
& \mathbb{E}[\tilde{L}(x, t+h)-\tilde{L}(x, t)]^{m} \\
& =\frac{1}{(2 \pi)^{m d}} \int_{\left(\mathbb{R}^{d}\right)^{m}} \int_{[t, t+h]^{m}} e^{-i \sum_{j=1}^{m}\left\langle u_{j}, x\right\rangle} \mathbb{E}\left[e^{i \sum_{j=1}^{m}\left\langle u_{j}, X_{t_{j}}\right\rangle}\right] \prod_{j=1}^{m} d t_{j} \prod_{j=1}^{m} d u_{j} \\
& =\frac{1}{(2 \pi)^{m d}} \int_{\left(\mathbb{R}^{d}\right)^{m}} \int_{[t, t+h]^{m}} e^{-i\left\langle v_{1}, x\right\rangle} \mathbb{E}\left[e^{i \sum_{j=1}^{m}\left\langle v_{j}, X_{t_{j}}-X_{t_{j-1}}\right\rangle}\right] \prod_{j=1}^{m} d t_{j} \prod_{j=1}^{m} d v_{j},
\end{aligned}
$$

where $t_{0}=0$ and the last equality in (2.3) (resp. (2.4) ) holds by the following changes of variables:

$$
u_{j}=v_{j}-v_{j+1}, \quad j=1, \cdots, m, \quad \text { with } \quad v_{m+1}=0 .
$$

For the purpose to estimate (2.3) and (2.4), we need first to estimate the characteristic function $\mathbb{E}\left[e^{i \sum_{j=1}^{m}\left\langle v_{j}, X_{t_{j}}-X_{t_{j-1}}\right\rangle}\right]$. Therefore, we introduce the following new condition, called $\alpha$-local nondeterminism ( $\alpha$-LND for short), which involves local estimation of the characteristic function of the increments:

Definition 2.4 Let $X=\left(X_{t}\right)_{t \in[0, T]}$ be a stochastic process with values in $\mathbb{R}^{d}$ and $J$ a subinterval of $[0, T] . X$ is said to be $\alpha-L N D$ on $J$, if for every nonnegative integers $m \geq 2$, and $k_{j, l}$, for $j=1, \cdots, m, l=1, \cdots, d$, there exist positive constants $c$ and $\varepsilon$, both may depend on $m$ and $k_{j, l}$, such that

$$
\left|\mathbb{E}\left[e^{i \sum_{j=1}^{m}\left\langle v_{j}, X_{t_{j}}-X_{t_{j-1}}\right\rangle}\right]\right| \leq \frac{c}{\prod_{j=1}^{m} \prod_{l=1}^{d}\left|v_{j, l}\right|^{k_{j, l}}\left(t_{j}-t_{j-1}\right)^{\alpha k_{j, l}}},
$$

for all $v_{j}=\left(v_{j, l} ; 1 \leq l \leq d\right) \in(\mathbb{R} \backslash\{0\})^{d}$, for $j=1, \cdots, m$, and for every ordered points $t_{1}<\cdots<t_{m}$ in $J$ with $t_{m}-t_{1}<\varepsilon$ and $t_{0}=0$. 
Remark 2.5 1. It is well-known that the local nondeterminism concept in the Gaussian context means that "the value of the process at a given time point is relatively unpredictable on the basis of a finite set of observations from the immediate past". In the Gaussian framework, Berman uses the conditional variance to express this. But unfortunately, he can't use the conditional variance beyond the Gaussian case because in a general framework the conditional variance is not deterministic. So, Berman has introduced the concept of local $g$-nondeterminism for general processes by replacing the incremental variance, which is a measure of local unpredictability, by a measure of local predictability, namely, the value of the incremental density function at the origin, see [7, Definition 5.1]. Hence, the local gnondeterminism concept reflects well his name. By the Fourier inversion theorem, it is easy to see that the condition in Definition 2.4 implies the local g-nondeterminism condition. On the other hand, Nolan has introduced the notion of characteristic function locally approximately independent increments (see [18, Definition 3.1]), which is equivalent in the Gaussian and stable framework to the classical LND condition. The condition in Definition 2.4 $(d=1)$ is an extension of Nolan's notion by replacing the characteristic functions $\left|\mathbb{E}\left[e^{i c_{m} u_{j}\left(X\left(t_{j}\right)-X\left(t_{j-1}\right)\right)}\right]\right|$ in the right-hand side of [18, Ineq. (3.3)] by $c\left|u_{j}\right|^{-k_{j}}\left(t_{j}-t_{j-1}\right)^{-\alpha k_{j}}$. For all these reasons, we choose to call the condition in Definition 2.4 by $\alpha$-local nondeterminism ( $\alpha$-LND).

2. Let $d=1$ and $Y=\left(Y_{t}\right)_{t \in[0, T]}$ be a centred Gaussian process that satisfies the classical local nondeterminism (LND) property on J. By [8, Lemma 2.3] we have for any $m \geq 2$, there exist two positive constants $c_{m}$ and $\varepsilon$ such that for every ordered points $t_{1}<\cdots<t_{m}$ in $J$ with $t_{m}-t_{1}<\varepsilon$, and $\left(v_{1}, \cdots, v_{m}\right) \in \mathbb{R}^{m} \backslash\{0\}$,

$$
\operatorname{Var}\left(\sum_{j=1}^{m} v_{j}\left(Y_{t_{j}}-Y_{t_{j-1}}\right)\right) \geq c_{m} \sum_{j=1}^{m} v_{j}^{2} \operatorname{Var}\left(Y_{t_{j}}-Y_{t_{j-1}}\right)
$$

Assume also that there exists a positive constant $K$, such that for every $s, t \in J$ with $s<t$,

$$
K(t-s)^{2 \alpha} \leq \operatorname{Var}\left(Y_{t}-Y_{s}\right)
$$

Hence $Y$ is $\alpha$-LND on $J$.

3. Let $d>1$ and $Y^{0}=\left(Y_{t}^{0}\right)_{t \in[0, T]}$ be a real-valued centred Gaussian process that verifies the classical local nondeterminism (LND) property on $J$ (i.e. (2.6) ) and (2.7). Define $Y_{t}=\left(Y_{t}^{1}, \cdots, Y_{t}^{d}\right)$, where $Y^{1}, \cdots, Y^{d}$ are independent copies of $Y^{0}$. Then $Y$ is $\alpha-L N D$ on $J$.

To end this section, we give the following theorem that explain clearly the fact that if a function's local time, $L(x, t)$, is Hölder continuous in $t$ uniformly in $x$, then this has a major effect on the Hölder continuity of the function itself. 
Theorem 2.6 (Theorem 8.7.1 in [1]) Let $\left(\theta_{s}\right)_{s \in[0, T]}$ be a continuous function with values in $\mathbb{R}^{d}$, possessing a local time, $L(x, t)$, satisfying: there exist positive constants $M$ and $\delta$, such that

$$
\sup _{x \in \mathbb{R}^{d}}|L(x, t)-L(x, s)| \leq M|t-s|^{\beta},
$$

for every $s, t \in[0, T]$ with $|t-s|<\delta$. Then all coordinate functions of $\theta$ are nowhere Hölder continuous of order greater than $(1-\beta) / d$.

\subsection{Classical Malliavin calculus}

In this section, we introduce some elements of Malliavin calculus, for more details we can refer to Nualart [19] (see also Sanz-Solé [20]). Let ( $W^{i}(t, x), t \in$ $[0, T], \quad x \in[0,1]), i=1, \cdots, d$, be $d$-independent space-time white noises defined on a probability space $(\Omega, \mathcal{F}, \mathbb{P})$, and set $W=\left(W^{1}, \cdots, W^{d}\right)$. For any $h=\left(h^{1}, \cdots, h^{d}\right) \in \mathcal{H}:=L^{2}\left([0, T] \times[0,1], \mathbb{R}^{d}\right)$, we put the Wiener integral $W(h)=\sum_{i=1}^{d} \int_{0}^{T} \int_{0}^{1} h^{i}(t, x) W^{i}(d x, d t)$. Denote by $\mathcal{S}$ the class of cylindrical random variables of the form $F=\varphi\left(W\left(h_{1}\right), \cdots, W\left(h_{n}\right)\right)$, with arbitrary $n \geq 1, h_{1}, \cdots, h_{n}$ in $\mathcal{H}$, and $\varphi \in C_{P}^{\infty}\left(\mathbb{R}^{n}\right)$, where $C_{P}^{\infty}\left(\mathbb{R}^{n}\right)$ is the set of realvalued functions $\varphi$ such that $\varphi$ and all its partial derivatives have at most polynomial growth. Let $F \in \mathcal{S}$, we define the derivative of $F$ as the $d$-dimensional stochastic process $D F=\left\{D_{t, x} F=\left(D_{t, x}^{(1)} F, \cdots, D_{t, x}^{(d)} F\right),(t, x) \in[0, T] \times[0,1]\right\}$, where, for $l=1, \cdots, d$,

$$
D_{t, x}^{(l)} F=\sum_{i=1}^{n} \frac{\partial \varphi}{\partial x_{i}}\left(W\left(h_{1}\right), \cdots, W\left(h_{n}\right)\right) h_{i}^{l}(t, x) .
$$

More generally set $D_{\alpha}^{k} F=D_{\alpha_{1}} \cdots D_{\alpha_{k}} F$ for the derivative of order $k$ of $F$, where $\alpha=\left(\alpha_{1}, \cdots, \alpha_{k}\right), \alpha_{i}=\left(t_{i}, x_{i}\right) \in[0, T] \times[0,1], k$ an integer. For any $p, k \geq 1$, we denote by $\mathbb{D}^{k, p}$ the closure of the class $\mathcal{S}$ with respect to the norm $\|\cdot\|_{k, p}$ defined by

$$
\|F\|_{k, p}=\left\{\mathbb{E}\left[|F|^{p}\right]+\sum_{j=1}^{k} \mathbb{E}\left[\left\|D^{j} F\right\|_{\mathcal{H} \otimes j}^{p}\right]\right\}^{\frac{1}{p}},
$$

where

$\left\|D^{j} F\right\|_{\mathcal{H}^{\otimes j}}=\left\{\sum_{i_{1}, \cdots, i_{j}=1}^{d} \int_{0}^{T} d t_{1} \int_{0}^{1} d x_{1} \cdots \int_{0}^{T} d t_{j} \int_{0}^{1} d x_{j}\left(D_{t_{1}, x_{1}}^{\left(i_{1}\right)} \cdots D_{t_{j}, x_{j}}^{\left(i_{j}\right)} F\right)^{2}\right\}^{\frac{1}{2}}$.

We put $\mathbb{D}^{\infty}=\cap_{p \geq 1} \cap_{k \geq 1} \mathbb{D}^{k, p}$. Let $0 \leq s<t \leq T$, we set $\mathcal{H}_{s, t}:=L^{2}([s, t] \times$ $\left.[0,1], \mathbb{R}^{d}\right)$. For any $F, G \in \mathbb{D}^{1, p}$ we point out that

$$
\langle D F, D G\rangle_{\mathcal{H}}=\sum_{l=1}^{d} \int_{0}^{T} \int_{0}^{1} D_{r, x}^{(l)} F D_{r, x}^{(l)} G d r d x
$$


and

$$
\langle D F, D G\rangle_{\mathcal{H}_{s, t}}=\sum_{l=1}^{d} \int_{s}^{t} \int_{0}^{1} D_{r, x}^{(l)} F D_{r, x}^{(l)} G d r d x .
$$

Let $\mathcal{V}$ be a separable Hilbert space. We define $\mathcal{S}_{\mathcal{V}}$ as the class of $\mathcal{V}$-valued smooth random variables of the form $u=\sum_{j=1}^{n} F_{j} v_{j}$, where $F_{j} \in \mathcal{S}$ and $v_{j} \in \mathcal{V}$. Similarly, we can introduce the analogous spaces $\mathbb{D}^{k, p}(\mathcal{V})$ and $\mathbb{D}^{\infty}(\mathcal{V})$, and the related norm $\|\cdot\|_{k, p, \mathcal{V}}$ defined by

$$
\|u\|_{k, p, \mathcal{V}}=\left\{\mathbb{E}\left[\|u\|_{\mathcal{V}}^{p}\right]+\sum_{j=1}^{k} \mathbb{E}\left[\left\|D^{j} u\right\|_{\mathcal{H} \otimes j \otimes \mathcal{V}}^{p}\right]\right\}^{\frac{1}{p}}
$$

We denote by $\delta$ the Skorohod integral, which is defined as the adjoint of the operator $D . \delta$ is an unbounded operator on $L^{2}(\Omega, \mathcal{H})$ taking values in $L^{2}(\Omega)$. The domain of $\delta$, denoted by $\operatorname{Dom}(\delta)$, is the set of $u \in L^{2}(\Omega, \mathcal{H})$ such that there exists a constant $c>0$ satisfying $\left|\mathbb{E}\left[\langle D F, u\rangle_{\mathcal{H}}\right]\right| \leq c\|F\|_{0,2}$, for every $F \in \mathbb{D}^{1,2}$. Let $u \in \operatorname{Dom}(\delta)$, then $\delta(u)$ is the unique element of $L^{2}(\Omega)$ characterized by the duality formula

$$
\mathbb{E}[F \delta(u)]=\mathbb{E}\left[\sum_{l=1}^{d} \int_{0}^{T} \int_{0}^{1} D_{t, x}^{(l)} F u_{l}(t, x) d t d x\right], \quad \text { for all } F \in \mathbb{D}^{1,2} .
$$

We will use the following estimate for the norm $\|\cdot\|_{k, p}$ of the Skorohod integral.

Proposition 2.7 ([19, Proposition 1.5.7) The divergence operator $\delta$ is continuous from $\mathbb{D}^{k+1, p}(\mathcal{H})$ to $\mathbb{D}^{k, p}$ for every $p>1, k \geq 0$. Therefore, there exists a constant $c_{k, p}>0$ such that for any $u \in \mathbb{D}^{k+1, p}(\mathcal{H})$,

$$
\|\delta(u)\|_{k, p} \leq c_{k, p}\|u\|_{k+1, p, \mathcal{H}}
$$

\subsection{Stochastic heat equation}

First note that Eq. (1.1) is formal, it can be formulated rigorously as follows (Walsh [21]): let $\mathcal{B}([0,1])$ be the Borel $\sigma$-algebra on $[0,1]$ and $W^{l}=$ $\left(W^{l}(t, A), \quad t \in[0, T], A \in \mathcal{B}([0,1])\right)$, where $l=1, \cdots, d$, be independent space-time white noises, defined on a complete probability space $(\Omega, \mathcal{F}, \mathbb{P})$, i.e, $W^{1}, \cdots, W^{d}$ are independent and $W^{l}$ is a centred Gaussian process with covariance function given by

$$
\mathbb{E}\left[W^{l}(t, A) W^{l}(s, B)\right]=(t \wedge s) \lambda(A \cap B),
$$

for $1 \leq l \leq d, t, s \in[0, T]$, and $A, B \in \mathcal{B}([0,1])$, where $\lambda$ is the Lebesgue measure. Set $W=\left(W^{1}, \cdots, W^{d}\right)$ and $W(t, x)=W(t,[0, x])$. For $t \in[0, T]$, let $\mathcal{F}_{t}=\sigma\{W(s, A), s \in[0, t], A \in \mathcal{B}([0,1])\} \vee \mathcal{N}$, where $\mathcal{N}$ is the collection of $\mathbb{P}$ null sets. We say that a process $u=\{u(t, x), t \in[0, T], x \in[0,1]\}$ is adapted 
to the filtration $\left(\mathcal{F}_{t}\right)_{0 \leq t \leq T}$ if $u(t, x)$ is $\mathcal{F}_{t}$-measurable for each $(t, x) \in[0, T] \times$ $[0,1]$. A mild solution of (1.1) is a jointly measurable $\mathbb{R}^{d}$-valued process $u=$ $\left(u_{1}, \cdots, u_{d}\right)$ such that $u$ is adapted to $\left(\mathcal{F}_{t}\right)_{0 \leq t \leq T}$ and for any $k \in\{1, \cdots, d\}$, $t \in[0, T]$, and $x \in[0,1]$,

$$
\begin{aligned}
u_{k}(t, x)= & \int_{0}^{t} \int_{0}^{1} G_{t-r}(x, v) \sum_{l=1}^{d} \sigma_{k, l}(u(r, v)) W^{l}(d r, d v) \\
& +\int_{0}^{t} \int_{0}^{1} G_{t-r}(x, v) b_{k}(u(r, v)) d v d r
\end{aligned}
$$

Here, The stochastic integral shall be read as in 21] and $G_{t}(x, y)$ denotes the Green kernel of the heat equation with Neumann boundary conditions (see [21, Chap. 3]). In this paper, We are not interested in the explicit form of $G_{t}(x, y)$, we will need just the following three properties:

- The symmetry [21, (3.6)]: $G_{t}(x, y)=G_{t}(y, x)$;

- The semi-group property [21, (3.6)]: $\int_{0}^{1} G_{t}(x, y) G_{s}(y, z) d y=G_{t+s}(x, z)$;

- The Gaussian-type bound [3, (A.1)]:

$$
c_{1} \phi_{t-s}(x-y) \leq G_{t-s}(x, y) \leq c_{2} \phi_{t-s}(x-y)
$$

where $\phi_{t-s}(x-y)=\frac{1}{\sqrt{2 \pi(t-s)}} \exp \left(-\frac{|x-y|^{2}}{2(t-s)}\right)$.

Modifying the results from 21 to the case $d \geq 1$, one can show that (when $\sigma_{k, l}$ and $b_{k}$ are Lipschitz), there exists a unique continuous $\mathbb{R}^{d}$-valued process $u=\{u(t, x), t \in[0, T], x \in[0,1]\}$ adapted to $\left(\mathcal{F}_{t}\right)_{0 \leq t \leq T}$ which is the mild solution of (1.1). Furthermore, it is shown in 2] that for any $0 \leq s \leq t \leq T$, $x, y \in[0,1]$, and $p>1$,

$$
\mathbb{E}\left[|u(t, x)-u(s, y)|^{p}\right] \leq C\left[|t-s|^{1 / 2}+|x-y|\right]^{p / 2} .
$$

Therefore, $u$ is $\left(\frac{1}{4}-\varepsilon\right)$-Hölder continuous in $t$ and $\left(\frac{1}{2}-\varepsilon\right)$-Hölder continuous in $x$.

Now we are concerned with the study of the Malliavin differentiability of $u$ and the equations fulfilled by its Malliavin derivatives (Proposition 2.8). We refer to Bally and Pardoux [3, Proposition 4.3, (4.16), (4.17)] for a complete proof in dimension one. In this paper, we work coordinate by coordinate, therefore the below proposition follows in the same way, and its proof is then omitted.

Proposition 2.8 (Proposition $4.1[9]$ ) Assume A1. Then for any $t \in[0, T]$ and $x \in[0,1]$ we have $u(t, x) \in\left(\mathbb{D}^{\infty}\right)^{d}$. Furthermore, its iterated derivative 
satisfies for $n \geq 1$ and all $r_{1}, \cdots, r_{n} \in[0, T]$ such that $r_{1} \vee \cdots \vee r_{n}<t$,

$$
\begin{aligned}
& D_{r_{1}, v_{1}}^{\left(i_{1}\right)} \cdots D_{r_{n}, v_{n}}^{\left(i_{n}\right)}\left(u_{k}(t, x)\right) \\
& =\sum_{p=1}^{d} G_{t-r_{p}}\left(x, v_{p}\right)\left(D_{r_{1}, v_{1}}^{\left(i_{1}\right)} \cdots D_{r_{p-1}, v_{p-1}}^{\left(i_{p-1}\right)} D_{r_{p+1}, v_{p+1}}^{\left(i_{p+1}\right)} \cdots D_{r_{n}, v_{n}}^{\left(i_{n}\right)}\left(\sigma_{k, l_{p}}\left(u\left(r_{p}, v_{p}\right)\right)\right)\right) \\
& \quad+\sum_{l=1}^{d} \int_{r_{1} \vee \cdots \vee r_{n}}^{t} \int_{0}^{1} G_{t-\tau}(x, z) \prod_{q=1}^{n} D_{r_{q}, v_{q}}^{\left(i_{q}\right)}\left(\sigma_{k, l}(u(\tau, z))\right) W^{l}(d \tau, d z) \\
& \quad+\int_{r_{1} \vee \cdots \vee r_{n}}^{t} \int_{0}^{1} G_{t-\tau}(x, z) \prod_{q=1}^{n} D_{r_{q}, v_{q}}^{\left(i_{q}\right)}\left(b_{k}(u(\tau, z))\right) d z d \tau,
\end{aligned}
$$

and when $t \leq r_{1} \vee \cdots \vee r_{n}$ we have $D_{r_{1}, v_{1}}^{\left(i_{1}\right)} \cdots D_{r_{n}, v_{n}}^{\left(i_{n}\right)}\left(u_{k}(t, x)\right)=0$. Finally, for any $p>1$,

$$
\sup _{(t, x) \in[0, T] \times[0,1]} \mathbb{E}\left[\left\|D^{n} u_{k}(t, x)\right\|_{\mathcal{H} \otimes n}^{p}\right]<\infty .
$$

Remark 2.9 Point out that, in particular, the first-order Malliavin derivative fulfils, for $r<t$,

$$
D_{r, v}^{(i)}\left(u_{k}(t, x)\right)=G_{t-r}(x, v) \sigma_{k, i}(u(r, v))+a_{k}(i, r, v, t, x),
$$

where

$$
\begin{aligned}
a_{k}(i, r, v, t, x)=\sum_{l=1}^{d} & \int_{r}^{t} \int_{0}^{1} G_{t-\tau}(x, z) D_{r, v}^{(i)}\left(\sigma_{k, l}(u(\tau, z))\right) W^{l}(d \tau, d z) \\
& +\int_{r}^{t} \int_{0}^{1} G_{t-\tau}(x, z) D_{r, v}^{(i)}\left(b_{k}(u(\tau, z))\right) d z d \tau
\end{aligned}
$$

and

$$
D_{r, v}^{(i)}\left(u_{k}(t, x)\right)=0 \quad \text { when } r>t .
$$

We conclude this section by the following useful lemma due to Morien [17. Lemma 4.2$]$ for $d=1$

Lemma 2.10 Assume A1. For all $q \geq 1, T>0$ there exists $C>0$ such that for all $0<\varepsilon \leq s \leq t \leq T$ and $0 \leq y \leq 1$,

$$
\sum_{i=1}^{d} \mathbb{E}\left[\left(\int_{s-\varepsilon}^{s} d r \int_{0}^{1} d v\left|\sum_{k=1}^{d}\right| D_{r, v}^{(i)}\left(u_{k}(t, y)\right)||^{2}\right)^{q}\right] \leq C \varepsilon^{q / 2}
$$




\section{Some new Malliavin calculus tools}

The main application of Malliavin calculus is the study of existence and smoothness of densities for the probability laws. For the purposes of the proof of our results, we introduce some new tools of Malliavin calculus that are interesting in the frame of general adapted processes. Let us first state the following definitions

Definition 3.1 For a fixed integer $n \geq 1$, let $F=\left(F_{1}, \cdots, F_{n}\right)$ be an $\mathbb{R}^{n \times d}$ valued random vector such that for $i=1, \cdots, n, F_{i}=\left(F_{i, 1}, \cdots, F_{i, d}\right)$ where $F_{i, k} \in \mathbb{D}^{1, p}$ for any $1 \leq i \leq n$ and $1 \leq k \leq d$. Let $\pi_{n}=\left(t_{1}, \cdots, t_{n}\right)$ with $0=t_{0}<t_{1}<\cdots<t_{n} \leq T$, we define the following matrices, for every $1 \leq i, j \leq n$,

$$
\Gamma^{i, j}=\left(\Gamma_{k, l}^{i, j}\right)_{1 \leq k, l \leq d} \quad \text { where } \quad \Gamma_{k, l}^{i, j}=\left\langle D F_{i, k}, D F_{j, l}\right\rangle_{\mathcal{H}_{t_{i-1}, t_{i}}}
$$

here $\mathcal{H}_{t_{i-1}, t_{i}}=L^{2}\left(\left[t_{i-1}, t_{i}\right] \times[0,1], \mathbb{R}^{d}\right)$. We write $\Gamma_{F}, \pi_{n}$ for the $\pi_{n}$-Malliavin matrix of $F$. That is, the following block matrix

$$
\Gamma_{F, \pi_{n}}=\left(\Gamma^{i, j}\right)_{1 \leq i, j \leq n}
$$

Remark 3.2 For an $\mathbb{R}^{d}$-valued adapted process $X=(X(t))_{t \in[0, T]}$ which is smooth in the Malliavin sense, we have the following known property:

$$
D_{r}^{(i)} X_{k}(t)=0 \quad \text { for any } r>t
$$

Hence, the $\pi_{n}$-Malliavin matrix of the vector of the increments of $X$ is a triangular block matrix. So, the calculus of its determinant is obvious, which explains the practical usefulness of considering the $\pi_{n}$-Malliavin matrices for adapted processes.

Definition 3.3 With the same notations as in Definition 3.1, $F$ is said to be $\pi_{n}$-nondegenerate, if it satisfies the following three conditions:

(i) For all $i=1, \cdots, n$, and $k=1, \cdots, d, F_{i, k} \in \mathbb{D}^{\infty}$.

(ii) $\Gamma_{F}, \pi_{n}$ is invertible a.s. and we denote by $\Gamma_{F, \pi_{n}}^{-1}$ its inverse.

(iii) $\left(\operatorname{det} \Gamma_{F, \pi_{n}}\right)^{-1} \in L^{p}$ for all $p \geq 1$.

The following integration by parts formula plays a crucial role in our paper.

Let $m \geq 1$. For any multi-index $\beta=\left(\beta_{1}, \cdots, \beta_{m}\right)$ with $\beta_{\theta}=\left(i_{\theta}, k_{\theta}\right) \in$ $\{1, \cdots, n\} \times\{1, \cdots, d\}$, for $\theta=1, \cdots, m$, we introduce the following notations:

$$
\partial_{\beta_{\theta}}:=\frac{\partial}{\partial x_{i_{\theta}, k_{\theta}}} \quad \text { for } \quad \theta=1, \cdots, m, \quad \text { and } \quad \partial_{\beta}:=\partial_{\beta_{1}} \cdots \partial_{\beta_{m}} .
$$


Proposition 3.4 For a fixed integer $n \geq 1$, let $\pi_{n}=\left(t_{1}, \cdots, t_{n}\right)$ with $0=$ $t_{0}<t_{1}<\cdots<t_{n} \leq T$ and $F=\left(F_{1}, \cdots, F_{n}\right)$ be a $\pi_{n}$-nondegenerate random vector with values in $\mathbb{R}^{n \times d}$ such that $F_{i}=\left(F_{i, 1}, \cdots, F_{i, d}\right)$, for $i=1, \cdots, n$. Let $G \in \mathbb{D}^{\infty}$ and let $g(x) \in C_{P}^{\infty}\left(\mathbb{R}^{n \times d}\right)$, where $x=\left(x_{i, k}, 1 \leq i \leq n, 1 \leq k \leq\right.$ $d)$. Then for all $m \geq 1$ and any multi-index $\beta=\left(\beta_{1}, \cdots, \beta_{m}\right)$, there exists $H_{\pi_{n}}^{\beta}(F, G) \in \mathbb{D}^{\infty}$ such that

$$
\mathbb{E}\left[\left(\partial_{\beta} g\right)(F) G\right]=\mathbb{E}\left[g(F) H_{\pi_{n}}^{\beta}(F, G)\right],
$$

where the random variables $H_{\pi_{n}}^{\beta}(F, G)$ are recursively given by

$$
\begin{gathered}
H_{\pi_{n}}^{(i, k)}(F, G)=\sum_{j=1}^{n} \sum_{l=1}^{d} \delta\left(G\left(\Gamma_{F, \pi_{n}}^{-1}\right)_{k, l}^{i, j} D F_{j, l} \mathbb{1}_{\left[t_{j-1}, t_{j}\right] \times[0,1]}\right), \\
H_{\pi_{n}}^{\beta}(F, G)=H_{\pi_{n}}^{\beta_{m}}\left(F, H_{\pi_{n}}^{\left(\beta_{1}, \cdots, \beta_{m-1}\right)}(F, G)\right) .
\end{gathered}
$$

Proof By the chain rule [19, Proposition 1.2.3] we have

$$
D g(F)=\sum_{j=1}^{n} \sum_{l=1}^{d} \frac{\partial g}{\partial x_{j, l}}(F) D F_{j, l} .
$$

Therefore, for every $i=1, \cdots, n$, and $k=1, \cdots, d$,

$$
\begin{aligned}
\left\langle D F_{i, k}, D g(F)\right\rangle_{\mathcal{H}_{t_{i-1}, t_{i}}} & =\sum_{j=1}^{n} \sum_{l=1}^{d} \frac{\partial g}{\partial x_{j, l}}(F)\left\langle D F_{i, k}, D F_{j, l}\right\rangle_{\mathcal{H}_{t_{i-1}, t_{i}}} \\
& =\sum_{j=1}^{n} \sum_{l=1}^{d} \frac{\partial g}{\partial x_{j, l}}(F) \Gamma_{k, l}^{i, j} .
\end{aligned}
$$

Hence, for any $i=1, \cdots, n$, and $k=1, \cdots, d$,

$$
\frac{\partial g}{\partial x_{i, k}}(F)=\sum_{j=1}^{n} \sum_{l=1}^{d}\left\langle D F_{j, l}, D g(F)\right\rangle_{\mathcal{H}_{t_{j-1}, t_{j}}}\left(\Gamma_{F, \pi_{n}}^{-1}\right)_{k, l}^{i, j} .
$$

And, consequently, we obtain

$$
G \frac{\partial g}{\partial x_{i, k}}(F)=\sum_{j=1}^{n} \sum_{l=1}^{d} G\left\langle D F_{j, l}, D g(F)\right\rangle_{\mathcal{H}_{t_{j-1}, t_{j}}}\left(\Gamma_{F, \pi_{n}}^{-1}\right)_{k, l}^{i, j} .
$$

On the other hand, we have for every $j=1, \cdots, n$, and $l=1, \cdots, d$,

$$
\left\langle D F_{j, l}, D g(F)\right\rangle_{\mathcal{H}_{t_{j-1}, t_{j}}}=\left\langle D F_{j, l} \mathbb{1}_{\left[t_{j-1}, t_{j}\right] \times[0,1]}, D g(F)\right\rangle_{\mathcal{H}} .
$$

Finally, taking expectations in (3.4) and using (3.5) and the duality formula (2.8) we get

$$
\mathbb{E}\left[\partial_{(i, k)} g(F) G\right]=\mathbb{E}\left[g(F) H_{\pi_{n}}^{(i, k)}(F, G)\right],
$$

where $H_{\pi_{n}}^{(i, k)}(F, G)$ is given by (3.2). The equation (3.3) follows by recurrence. 
The below lemma will be devoted to get some estimations of the $\|\cdot\|_{k, p}$-norm of elements of the Malliavin matrix

Lemma 3.5 Let $0 \leq s<t \leq T$ and $F, G \in \mathbb{D}^{\infty}$, then we have the following

$$
\left\|\langle D F, D G\rangle_{\mathcal{H}_{s, t}}\right\|_{k, p} \leq C\|D F\|_{k, 2 p, \mathcal{H}}\|D G\|_{k, 2 p, \mathcal{H}}
$$

Proof By definition, we have

$$
\begin{aligned}
\left\|\langle D F, D G\rangle_{\mathcal{H}_{s, t}}\right\|_{k, p}= & \left\{\mathbb{E}\left[\left|\langle D F, D G\rangle_{\mathcal{H}_{s, t}}\right|^{p}\right]\right. \\
& \left.+\sum_{j=1}^{k} \mathbb{E}\left[\left\|D^{j}\langle D F, D G\rangle_{\mathcal{H}_{s, t}}\right\|_{\mathcal{H}^{\otimes j}}^{p}\right]\right\}^{\frac{1}{p}} .
\end{aligned}
$$

We can easily check that

$$
\mathbb{E}\left[\left|\langle D F, D G\rangle_{\mathcal{H}_{s, t}}\right|^{p}\right] \leq\|D F\|_{k, 2 p, \mathcal{H}}^{p}\|D G\|_{k, 2 p, \mathcal{H}}^{p}
$$

On the other hand, we get for $j \geq 1$ and $p>2$,

$$
\begin{aligned}
& E\left[\left\|D^{j}\langle D F, D G\rangle_{\mathcal{H}_{s, t}}\right\|_{\mathcal{H}^{\otimes j}}^{p}\right] \\
& =E\left[\left\|D^{j}\left(\int_{s}^{t} \int_{0}^{1} D_{r, x} F \cdot D_{r, x} G d r d x\right)\right\|_{\mathcal{H}^{\otimes j}}^{p}\right] \\
& =\mathbb{E}\left[\left\{\sum_{i_{1}, \cdots, i_{j}=1}^{d} \int_{0}^{T} d r_{1} \int_{0}^{1} d x_{1} \cdots \int_{0}^{T} d r_{j} \int_{0}^{1} d x_{j}\right.\right. \\
& \left.\left.\left|\sum_{l=1}^{d} \int_{s}^{t} \int_{0}^{1} D_{r_{1}, x_{1}}^{\left(i_{1}\right)} \cdots D_{r_{j}, x_{j}}^{\left(i_{j}\right)}\left(D_{r, x}^{(l)} F \cdot D_{r, x}^{(l)} G\right) d r d x\right|^{2}\right\}^{\frac{p}{2}}\right]
\end{aligned}
$$

Let $J=\left\{i_{1}, \cdots, i_{j}\right\}$ and $I \subset\left\{i_{1}, \cdots, i_{j}\right\}$ such that $I=\left\{i_{k_{1}}, \cdots, i_{k_{m}}\right\}$, and $\alpha_{I}=\left(t_{k_{1}}, x_{k_{1}}, \cdots, t_{k_{m}}, x_{k_{m}}\right)$, we put $D_{\alpha_{I}}^{I} F:=D_{t_{k_{1}}, x_{k_{1}}}^{\left(i_{k_{1}}\right)} \cdots D_{t_{k_{m}}, x_{k_{m}}}^{\left(i_{k_{m}}\right)} F$. When $I=\varnothing$, we set $D^{I} F=F$. Then we have for $F, G \in \mathbb{D}^{\infty}$ and $\alpha=$ $\left(t_{1}, x_{1}, \cdots, t_{j}, x_{j}\right)$, the following Leibniz's rule

$$
D_{\alpha}^{J}(F \cdot G)=\sum_{I \subset\left\{i_{1}, \cdots, i_{j}\right\}} D_{\alpha_{I}}^{I} F \cdot D_{\alpha_{I^{c}}}^{I^{c}} G
$$


where $I^{c}$ is the complement of $I$. We denote by $|I|$ the cardinality of $I$. Combining (3.9) and (3.10), we get

$$
\begin{aligned}
& E\left[\left\|D^{j}\langle D F, D G\rangle_{\mathcal{H}_{s, t}}\right\|_{\mathcal{H}^{\otimes j}}^{p}\right] \\
& =\mathbb{E}\left[\left\{\sum_{i_{1}, \cdots, i_{j}=1}^{d} \int_{0}^{T} d r_{1} \int_{0}^{1} d x_{1} \cdots \int_{0}^{T} d r_{j} \int_{0}^{1} d x_{j}\right.\right. \\
& \left.\left.\left|\sum_{l=1}^{d} \sum_{I \subset\left\{i_{1}, \cdots, i_{j}\right\}} \int_{s}^{t} \int_{0}^{1} D_{\alpha_{I}}^{I} D_{r, x}^{(l)} F \cdot D_{\alpha_{I^{c}}}^{I^{c}} D_{r, x}^{(l)} G d r d x\right|^{2}\right\}^{\frac{p}{2}}\right] .
\end{aligned}
$$

Therefore, by the convexity, Hölder's inequality, and Fubini's theorem, we obtain that this last term is less than or equal to

$$
\begin{aligned}
& \hat{C} \sum_{i_{1}, \cdots, i_{j}=1}^{d} \sum_{l=1}^{d} \sum_{I \subset\left\{i_{1}, \cdots, i_{j}\right\}} \mathbb{E}\left[\left\{\int_{([0, T] \times[0,1])^{|I|}} d \alpha_{I} \int_{0}^{T} d r \int_{0}^{1} d x\left|D_{\alpha_{I}}^{I} D_{r, x}^{(l)} F\right|^{2}\right\}^{p}\right]^{\frac{1}{2}} \\
& \cdot \mathbb{E}\left[\left\{\int_{([0, T] \times[0,1])^{\left|I^{c}\right|}} d \alpha_{I^{c}} \int_{0}^{T} d r \int_{0}^{1} d x\left|D_{\alpha_{I^{c}}}^{I^{c}} D_{r, x}^{(l)} G\right|^{2}\right\}^{p}\right]^{\frac{1}{2}} \\
& \leq \hat{C} \sum_{i_{1}, \cdots, i_{j}=1}^{d} \sum_{l=1}^{d} \sum_{I \subset\left\{i_{1}, \cdots, i_{j}\right\}} \mathbb{E}\left[\left\|D^{|I|+1} F\right\|_{\mathcal{H} \otimes(|I|+1)}^{2 p} \cdot \mathbb{E}\left[\left\|D^{\left|I^{c}\right|+1} G\right\|_{\mathcal{H} \otimes\left(\left|I^{c}\right|+1\right)}^{2 p}\right]^{\frac{1}{2}}\right. \\
& \leq \hat{C} \sum_{i_{1}, \cdots, i_{j}=1}^{d} \sum_{l=1}^{d} \sum_{I \subset\left\{i_{1}, \cdots, i_{j}\right\}}\|D F\|_{k, 2 p, \mathcal{H}}^{p}\|D G\|_{k, 2 p, \mathcal{H}}^{p} \leq C\|D F\|_{k, 2 p, \mathcal{H}}^{p}\|D G\|_{k, 2 p, \mathcal{H}}^{p} .
\end{aligned}
$$

One can easily derive from (3.7), (3.8), and (3.11) the inequality (3.6).

The next lemma gives a sharp estimate of the $\|\cdot\|_{0,2}$-norm of the random variables $H_{\pi_{n}}^{\beta}(F, G)$

Lemma 3.6 For a fixed integer $n \geq 1$, let $\pi_{n}=\left(t_{1}, \cdots, t_{n}\right)$ with $0=t_{0}<$ $t_{1}<\cdots<t_{n} \leq T$ and $F=\left(F_{1}, \cdots, F_{n}\right)$ be a $\pi_{n}$-nondegenerate random vector with values in $\mathbb{R}^{n \times d}$ such that, $F_{i}=\left(F_{i, 1}, \cdots, F_{i, d}\right)$, for $i=1, \cdots, n$. Let $\beta=$ $\left(\beta_{1}, \cdots, \beta_{m}\right)$ with $\beta_{\theta}=\left(i_{\theta}, k_{\theta}\right) \in\{1, \cdots, n\} \times\{1, \cdots, d\}$, for $\theta=1, \cdots, m$, then there exists a constant $C>0$ such that

$$
\begin{aligned}
&\left\|H_{\pi_{n}}^{\beta}(F, 1)\right\|_{0,2} \leq C\left\|\left(\operatorname{det} \Gamma_{F}, \pi_{n}\right)^{-1}\right\|_{m, 2^{m+2}}^{m} \\
& \cdot \prod_{\theta=1}^{m}\left\|D F_{i_{\theta}, k_{\theta}}\right\|_{m, 2^{2(m+n d)}, \mathcal{H}} \prod_{\left(i_{0}, k_{0}\right) \in O_{\left(i_{\theta}, k_{\theta}\right)}}\left\|D F_{i_{0}, k_{0}}\right\|_{m, 2^{2(m+n d)}, \mathcal{H}}^{2},
\end{aligned}
$$

where $O_{\left(i_{\theta}, k_{\theta}\right)}=\left\{\left(i_{0}, k_{0}\right) \in\{1, \cdots, n\} \times\{1, \cdots, d\} ;\left(i_{0}, k_{0}\right) \neq\left(i_{\theta}, k_{\theta}\right)\right\}$. 
Proof By (3.3) and (3.2), we write

$$
\begin{aligned}
& \left\|H_{\pi_{n}}^{\beta}(F, 1)\right\|_{0,2} \\
& =\left\|H_{\pi_{n}}^{\beta_{m}}\left(F, H_{\pi_{n}}^{\left(\beta_{1}, \cdots, \beta_{m-1}\right)}(F, 1)\right)\right\|_{0,2} \\
& =\left\|\sum_{j=1}^{n} \sum_{l=1}^{d} \delta\left(H_{\pi_{n}}^{\left(\beta_{1}, \cdots, \beta_{m-1}\right)}(F, 1)\left(\Gamma_{F, \pi_{n}}^{-1}\right)_{k_{m}, l}^{i_{m}, j} D F_{j, l} \mathbb{1}_{\left[t_{j-1}, t_{j}\right] \times[0,1]}\right)\right\|_{0,2} .
\end{aligned}
$$

According to (2.9), and Hölder's inequality for the Malliavin norms (cf. 22, Proposition 1.10]), we obtain that this last term is less than or equal to

$$
\begin{aligned}
& C\left\|H_{\pi_{n}}^{\left(\beta_{1}, \cdots, \beta_{m-1}\right)}(F, 1)\right\|_{1,2^{2}} \sum_{j=1}^{n} \sum_{l=1}^{d}\left\|\left(\Gamma_{F, \pi_{n}}^{-1}\right)_{k_{m}, l}^{i_{m}, j}\right\|_{1,2^{3}}\left\|D F_{j, l} \mathbb{1}_{\left[t_{j-1}, t_{j}\right] \times[0,1]}\right\|_{1,2^{3}, \mathcal{H}} \\
& \leq C\left\|H_{\pi_{n}}^{\left(\beta_{1}, \cdots, \beta_{m-1}\right)}(F, 1)\right\|_{1,2^{2}} \sum_{j=1}^{n} \sum_{l=1}^{d}\left\|\left(\Gamma_{F, \pi_{n}}^{-1}\right)_{k_{m}, l}^{i_{m}, j}\right\|_{1,2^{3}}\left\|D F_{j, l}\right\|_{1,2^{3}, \mathcal{H}} \cdot
\end{aligned}
$$

On the other hand, we know that the inverse of the matrix $\Gamma_{F}, \pi_{n}$ is the transpose of its cofactor matrix, that we denote by $A_{F, \pi_{n}}$, multiplied by the inverse of its determinant i.e.,

$$
\Gamma_{F, \pi_{n}}^{-1}=\frac{1}{\operatorname{det} \Gamma_{F, \pi_{n}}} A_{F, \pi_{n}}^{\prime}
$$

where $A_{F, \pi_{n}}^{\prime}$ is the transpose of $A_{F}, \pi_{n}$. Set $N=\{1, \cdots, n\}$ and $D=\{1, \cdots, d\}$. Let $B(i, k ; j, l)=\left(b\left(i_{0}, k_{0} ; j_{0}, l_{0}\right)\right)$, where $\left(i_{0}, k_{0}\right),\left(j_{0}, l_{0}\right) \in O:=\{(p, q) \in$ $N \times D ;(p, q) \neq(n, d)\}$, be the $(n \times d-1) \times(n \times d-1)$-matrix obtained by removing from $\Gamma_{F}, \pi_{n}$ its row $(i, k)$ and column $(j, l)$. Let $O_{(i, k)}$ be the set of $\left(i_{0}, k_{0}\right) \in N \times D$ such that $\left(i_{0}, k_{0}\right) \neq(i, k), O_{(j, l)}$ the set of $\left(j_{0}, l_{0}\right) \in N \times D$ with $\left(j_{0}, l_{0}\right) \neq(j, l), \Xi=\{\eta ; \eta$ permutation of $O\}$, and $\Pi:=\left\{\varrho ; \varrho: O_{(i, k)} \rightarrow\right.$ $O_{(j, l)}$ bijective map $\}$, hence by Hölder's inequality for the Malliavin norms and Lemma 3.5 we have

$$
\begin{aligned}
\left\|\left(A_{F, \pi_{n}}\right)_{k, l}^{i, j}\right\|_{1,2^{4}} & =\|\operatorname{det}(B(i, k ; j, l))\|_{1,2^{4}} \\
& =\left\|\sum_{\eta \in \Xi} \varepsilon(\eta) \prod_{(p, q) \in O} b(p, q ; \eta(p, q))\right\|_{1,2^{4}} \\
& \leq \sum_{\eta \in \Xi} \prod_{(p, q) \in O}\|b(p, q ; \eta(p, q))\|_{1,2^{n d+2}} \\
& =\sum_{\varrho \in \Pi} \prod_{\left(i_{0}, k_{0}\right) \in O_{(i, k)}}\left\|\left\langle D F_{i_{0}, k_{0}}, D F_{\varrho\left(i_{0}, k_{0}\right)}\right\rangle_{\mathcal{H}_{t_{i_{0}-1, t_{i}}}}\right\|_{1,2^{n d+2}}\left\|D F_{i_{0}, k_{0}}\right\|_{1,2^{2(n d)+4}, \mathcal{H}}\left\|D F_{\varrho\left(i_{0}, k_{0}\right)}\right\|_{1,2^{2(n d)+4}, \mathcal{H}} .
\end{aligned}
$$


According to (3.14), (3.15), and Hölder's inequality for the Malliavin norms, we get

$$
\begin{aligned}
\left\|\left(\Gamma_{F, \pi_{n}}^{-1}\right)_{k_{m}, l}^{i_{m}, j}\right\|_{1,2^{3}} & \leq C\left\|\left(\operatorname{det} \Gamma_{F, \pi_{n}}\right)^{-1}\right\|_{1,2^{4}} \\
& \cdot \sum_{\mu \in \Pi_{m}} \prod_{\left(j_{0}, l_{0}\right) \in O_{(j, l)}}\left\|D F_{j_{0}, l_{0}}\right\|_{1,2^{2 n d+4}, \mathcal{H}}\left\|D F_{\mu\left(j_{0}, l_{0}\right)}\right\|_{1,2^{2 n d+4}, \mathcal{H}},
\end{aligned}
$$

where $\Pi_{m}:=\left\{\mu ; \mu: O_{(j, l)} \rightarrow O_{\left(i_{m}, k_{m}\right)}\right.$ bijective map $\}$ and $O_{\left(i_{m}, k_{m}\right)}$ is the set of $\left(i_{0}, k_{0}\right) \in N \times D$ such that $\left(i_{0}, k_{0}\right) \neq\left(i_{m}, k_{m}\right)$. We derive from (3.16) that

$$
\begin{aligned}
& \left\|\left(\Gamma_{F, \pi_{n}}^{-1}\right)_{k_{m}, l}^{i_{m}, j}\right\|_{1,2^{3}} \\
& \leq C\left\|\left(\operatorname{det} \Gamma_{F, \pi_{n}}\right)^{-1}\right\|_{1,2^{4}} \sum_{\mu \in \Pi_{m}} \prod_{\left(j_{0}, l_{0}\right) \in O_{(j, l)}}\left\|D F_{j_{0}, l_{0}}\right\|_{1,2^{2 n d+4}, \mathcal{H}} \\
& \cdot \prod_{\left(i_{0}, k_{0}\right) \in O_{\left(i_{m}, k_{m}\right)}}\left\|D F_{i_{0}, k_{0}}\right\|_{1,2^{2 n d+4}, \mathcal{H}} \\
& =C\left|\Pi_{m}\right|\left\|\left(\operatorname{det} \Gamma_{F, \pi_{n}}\right)^{-1}\right\|_{1,2^{4}}\left\|D F_{j, l}\right\|_{1,2^{2 n d+4}, \mathcal{H}}\left\|D F_{i_{m}, k_{m}}\right\|_{1,2^{2 n d+4}, \mathcal{H}} \\
& \cdot \prod_{\left(i_{0}, k_{0}\right) \in O_{(j, l)} \cap O_{\left(i_{m}, k_{m}\right)}}\left\|D F_{i_{0}, k_{0}}\right\|_{1,2^{2 n d+4}, \mathcal{H}}^{2},
\end{aligned}
$$

where $\left|\Pi_{m}\right|$ is the cardinality of $\Pi_{m}$. Combining (3.13) and (3.17), we obtain

$$
\begin{aligned}
& \left\|H_{\pi_{n}}^{\beta}(F, 1)\right\|_{0,2} \\
& \leq C_{1}\left\|H_{\pi_{n}}^{\left(\beta_{1}, \cdots, \beta_{m-1}\right)}(F, 1)\right\|_{1,2^{2}}\left\|\left(\operatorname{det} \Gamma_{F}, \pi_{n}\right)^{-1}\right\|_{1,2^{4}}\left\|D F_{i_{m}, k_{m}}\right\|_{1,2^{2 n d+4}, \mathcal{H}} \\
& \cdot \sum_{j=1}^{n} \sum_{l=1}^{d}\left\|D F_{j, l}\right\|_{1,2^{2 n d+4}, \mathcal{H}} \prod_{\left(i_{0}, k_{0}\right) \in O_{(j, l)} \cap O_{\left(i_{m}, k_{m}\right)}}\left\|D F_{i_{0}, k_{0}}\right\|_{1,2^{2 n d+4}, \mathcal{H}}^{2}\left\|D F_{j, l}\right\|_{1,2^{3}, \mathcal{H}} \\
& \leq C_{2}\left\|H_{\pi_{n}}^{\left(\beta_{1}, \cdots, \beta_{m-1}\right)}(F, 1)\right\|_{1,2^{2}}\left\|\left(\operatorname{det} \Gamma_{F, \pi_{n}}\right)^{-1}\right\|_{1,2^{4}}\left\|D F_{i_{m}, k_{m}}\right\|_{1,2^{2 n d+4}, \mathcal{H}} \\
& \cdot \prod_{\left.\left(i_{0}, k_{0}\right) \in O_{\left(i_{m}, k_{m}\right)}\right)}\left\|D F_{i_{0}, k_{0}}\right\|_{1,2^{2 n d+4}, \mathcal{H}}^{2}
\end{aligned}
$$

Finally, by recurrence on $m$ we get the inequality (3.12), which finishes the proof of Lemma 3.6

Now we will state the criterion for smoothness of density for a random vector which is $\pi_{n}$-nondegenerate, and give the formula of its derivatives. The proof is similar to [19, Theorem 2.1.4].

Theorem 3.7 For a fixed integer $n \geq 1$, let $\pi_{n}=\left(t_{1}, \cdots, t_{n}\right)$ with $0=t_{0}<$ $t_{1}<\cdots<t_{n} \leq T$ and $F=\left(F_{1}, \cdots, F_{n}\right)$ be a $\pi_{n}$-nondegenerate random vector 
with values in $\mathbb{R}^{n \times d}$ such that $F_{i}=\left(F_{i, 1}, \cdots, F_{i, d}\right)$, for $i=1, \cdots, n$. Then $F$ possesses a density $p_{\pi_{n}}(x)$, where $x=\left(x_{i, k} ; 1 \leq i \leq n, 1 \leq k \leq d\right) \in \mathbb{R}^{n \times d}$, which is infinitely differentiable and given by

$$
p_{\pi_{n}}(x)=\mathbb{E}\left[\mathbb{1}_{\{F>x\}} H_{\pi_{n}}^{\gamma}(F, 1)\right],
$$

where $\gamma=((i, k) ; 1 \leq i \leq n, 1 \leq k \leq d)$. Fix $m \geq 1$. For any multi-index $\beta=$ $\left(\beta_{1}, \cdots, \beta_{m}\right)$ with $\beta_{\theta}=\left(i_{\theta}, k_{\theta}\right) \in\{1, \cdots, n\} \times\{1, \cdots, d\}$, for $\theta=1, \cdots, m$, we have

$$
\partial_{\beta} p_{\pi_{n}}(x)=(-1)^{m} \mathbb{E}\left[\mathbb{1}_{\{F>x\}} H_{\pi_{n}}^{(\beta, \gamma)}(F, 1)\right],
$$

where $\mathbb{1}_{\{F>x\}}:=\prod_{i=1}^{n} \prod_{k=1}^{d} \mathbb{1}_{\left\{F_{i, k}>x_{i, k}\right\}}$.

\section{Proof of Theorem 1.3}

Our purpose in this section is to establish the Gaussian-type lower bound for the density of $u(t, x)-u(s, x)$, when $0 \leq s<t \leq T$, and the upper bound of Gaussian-type for the partial derivatives of the density of $\left(u\left(t_{1}, x\right)-\right.$ $\left.u\left(t_{0}, x\right), \cdots, u\left(t_{n}, x\right)-u\left(t_{n-1}, x\right)\right)$, where $0=t_{0}<t_{1}<\cdots<t_{n} \leq T$.

4.1 The Gaussian-type lower bound

The proof of Theorem 1.3(a) is quite similar to that in [9, Section 5] (see also [14, Theorem 10] for the original work in dimension $d=1$ ). Thus we will only sketch the main ideas.

Remark 4.1 For clarity reasons, we borrow most of the notations in this subsection from [9, Section 5] and [14]. Therefore, the notations used here are independent of the rest of this paper.

Proof (Proof of Theorem 1.3(a)) Let $x \in(0,1)$ and $0 \leq s<t \leq T$, the proof of Theorem 1.3(a) follows the same lines as in [9, Section 5] (or [14] for $d=1$ ). We only sketch the main points where there is a difference between the chose of $F=u(t, x)$ (the study of [9]) and $F=u(t, x)-u(s, x)$. The idea of KohatsuHiga [14] is to show that $u(t, x)-u(s, x)$ is a $d$-dimensional uniformly elliptic random vector and therefore apply [14, Theorem 5].

Set $g(r, v):=G_{t-r}(x, v)$. Let us consider a sufficiently fine partition $\{s=$ $\left.t_{0}<\cdots<t_{N}=t\right\}$. By the properties of $G$, i.e., symmetry, semi-group property and, Gaussian-type bound, there exist two positive constants $c_{1}$ and $c_{2}$ such that

$$
c_{1}|t-s|^{1 / 4} \leq\|g\|_{L^{2}([s, t] \times[0,1])} \leq c_{2}|t-s|^{1 / 4} .
$$

Let

$$
\tilde{F}_{n}^{i}=F_{n}^{i}-u_{i}(s, x),
$$


where for $1 \leq i \leq d$ and $0 \leq n \leq N, F_{n}^{i}$ are given as in [9, Section 5] by

$$
\begin{aligned}
F_{n}^{i}=\int_{0}^{t_{n}} & \int_{0}^{1} G_{t-r}(x, v) \sum_{j=1}^{d} \sigma_{i j}(u(r, v)) W^{j}(d r, d v) \\
& +\int_{0}^{t_{n}} \int_{0}^{1} G_{t-r}(x, v) b_{i}(u(r, v)) d v d r .
\end{aligned}
$$

Point out that $\tilde{F}_{n}^{i} \in \mathcal{F}_{t_{n}}$. We will need the following lemma.

Lemma 4.2 We assume $\mathbf{A} 1$ and A2. Then

(1) $\left\|\tilde{F}_{n}^{i}\right\|_{k, p} \leq c_{k, p}, \quad 1 \leq i \leq d$;

(2) $\left\|\left(\operatorname{det} \gamma_{\tilde{F}_{n}}\left(t_{n-1}\right)\right)^{-1}\right\|_{p, t_{n-1}} \leq c_{p}\left(\Delta_{n-1}(g)\right)^{-d}:=c_{p}\left(\|g\|_{L^{2}\left(\left[t_{n-1}, t_{n}\right] \times[0,1]\right)}^{2}\right)^{-d}$, where $\|\cdot\|_{p, t_{n-1}}$ denotes the conditional $L^{p}$-norm and $\gamma_{\tilde{F}_{n}}\left(t_{n-1}\right)$ is the conditional Malliavin matrix of $\tilde{F}_{n}$ given $\mathcal{F}_{t_{n}}$.

Proof (Proof of Lemma 4.2) The point (1) is a consequence of 9, Lemma 5.1(i)] and (2.13). Otherwise, by the fact that $s \leq t_{n-1}$ and (2.16), we have the following:

$$
\gamma_{\tilde{F}_{n}}\left(t_{n-1}\right)=\gamma_{F_{n}}\left(t_{n-1}\right)
$$

where $\gamma_{F_{n}}\left(t_{n-1}\right)$ is the conditional Malliavin matrix of $F_{n}$ given $\mathcal{F}_{t_{n}}$. Then we can conclude the proof of (2) by [9, Lemma 5.1(ii)] or [14, Lemma 7] (the last reference is for $d=1$, but the same ideas, in the proof, still work for $d>1)$.

Continuing the proof of Theorem 1.3(a). We remark that

$$
\tilde{F}_{n}^{i}-\tilde{F}_{n-1}^{i}=F_{n}^{i}-F_{n-1}^{i} .
$$

Hence, in order to get the expansion of $\tilde{F}_{n}^{i}-\tilde{F}_{n-1}^{i}$ as in [14, Lemma 9], one has to obtain that expansion (i.e., as in [14, Lemma 9]) for $F_{n}^{i}-F_{n-1}^{i}$. The remainder of the proof is the same as in [9, Section 5].

4.2 The Gaussian-type upper bound for the partial derivatives of the density

Our aim in this subsection is to prove Theorem 1.3(b). Let $p_{\pi_{n}, x}(\xi)$ be the joint density of the $\mathbb{R}^{n \times d}$-valued random vector

$$
Z=\left(u\left(t_{1}, x\right)-u\left(t_{0}, x\right), \cdots, u\left(t_{n}, x\right)-u\left(t_{n-1}, x\right)\right),
$$

where $u\left(t_{i}, x\right)-u\left(t_{i-1}, x\right)=\left(u_{1}\left(t_{i}, x\right)-u_{1}\left(t_{i-1}, x\right), \cdots, u_{d}\left(t_{i}, x\right)-u_{d}\left(t_{i-1}, x\right)\right)$, $\xi=\left(\xi_{i, k} ; 1 \leq i \leq n, 1 \leq k \leq d\right) \in \mathbb{R}^{n \times d}, x \in(0,1)$, and $\pi_{n}=\left(t_{1}, \cdots, t_{n}\right)$ with $0=t_{0}<t_{1}<\cdots<t_{n} \leq T$. The existence of this joint density, which is infinitely differentiable, will be a consequence of Theorem 3.7 Proposition 2.8, and Theorem 4.5 .

The following proposition gives an upper bound for the Malliavin norm of the derivative of the increments of the process $\{u(t, x), t \in[0, T]\}$. 
Proposition 4.3 (Proposition 6.2 in [9]) Assume A1. Then for any $0 \leq$ $s \leq t \leq T, x \in[0,1], p>1$, and $m \geq 1$,

$$
\mathbb{E}\left[\left\|D^{m}\left(u_{k}(t, x)-u_{k}(s, x)\right)\right\|_{\mathcal{H} \otimes m}^{p}\right] \leq C_{T}|t-s|^{p / 4}, \quad k=1, \cdots, d .
$$

Now we will investigate the $\pi_{n}$-Malliavin matrix, $\Gamma_{Z}, \pi_{n}$, of $Z$ ( $Z$ is given by (4.1)). Note that $\Gamma_{Z, \pi_{n}}=\left(\Gamma^{i, j}\right)_{1 \leq i, j \leq n}$ is the random block matrix, where $\Gamma^{i, j}=\left(\Gamma_{k, l}^{i, j}\right)_{1 \leq k, l \leq d}$ and the $\Gamma_{k, l}^{i, j}$ are given by

$$
\Gamma_{k, l}^{i, j}=\left\langle D\left(u_{k}\left(t_{i}, x\right)-u_{k}\left(t_{i-1}, x\right)\right), D\left(u_{l}\left(t_{j}, x\right)-u_{l}\left(t_{j-1}, x\right)\right)\right\rangle_{\mathcal{H}_{t_{i-1}, t_{i}}},
$$

here $1 \leq i, j \leq n, 1 \leq k, l \leq d$, and $\mathcal{H}_{t_{i-1}, t_{i}}=L^{2}\left(\left[t_{i-1}, t_{i}\right] \times[0,1], \mathbb{R}^{d}\right)$. The matrix $\Gamma_{Z, \pi_{n}}$ is not a symmetric matrix, in general, (but the matrices $\Gamma^{i, i}$, for $i=1, \cdots, n$, are symmetric). Based on the formula (2.16), we get the following key remark

Remark 4.4 $\Gamma_{Z, \pi_{n}}$ is a triangular block matrix almost surly, i.e., for all $1 \leq i, j \leq n$ with $j<i$, we have $\Gamma^{i, j} \equiv 0$ a.s.

A consequence of the above remark is that

$$
\operatorname{det}\left(\Gamma_{Z, \pi_{n}}\right)=\prod_{i=1}^{n} \operatorname{det}\left(\Gamma^{i, i}\right) \quad \text { a.s. }
$$

The below theorem gives an estimate on the Malliavin norm of the determinant of the inverse of the matrix $\Gamma_{Z}, \pi_{n}$.

Theorem 4.5 Assume A1 and A2. Let $\pi_{n}=\left(t_{1}, \cdots, t_{n}\right)$ with $0=t_{0}<t_{1}<$ $\cdots<t_{n} \leq T, x \in(0,1)$, and $Z$ given by (4.1), then for any $k \geq 0, p>1$,

$$
\left\|\left(\operatorname{det} \Gamma_{Z, \pi_{n}}\right)^{-1}\right\|_{k, p} \leq K \prod_{i=1}^{n}\left(t_{i}-t_{i-1}\right)^{-d / 2},
$$

where $K$ is a positive constant.

Proof By (4.3) and Hölder's inequality for the Malliavin norms (cf. [22, Proposition 1.10]), we get

$$
\left\|\left(\operatorname{det} \Gamma_{Z, \pi_{n}}\right)^{-1}\right\|_{k, p} \leq \prod_{i=1}^{n}\left\|\left(\operatorname{det} \Gamma^{i, i}\right)^{-1}\right\|_{k, 2^{n-1} p} .
$$

Let $\tilde{p}=2^{n-1} p$. By definition, we have

$$
\begin{aligned}
\left\|\left(\operatorname{det} \Gamma^{i, i}\right)^{-1}\right\|_{k, \tilde{p}}=\left\{\mathbb{E}\left[\left|\left(\operatorname{det} \Gamma^{i, i}\right)^{-1}\right|^{\tilde{p}}\right]\right. \\
\\
\left.+\sum_{l=1}^{k} \mathbb{E}\left[\left\|D^{l}\left(\operatorname{det} \Gamma^{i, i}\right)^{-1}\right\|_{\mathcal{H}^{\otimes l}}^{\tilde{p}}\right]\right\}^{\frac{1}{\tilde{p}}} .
\end{aligned}
$$


To estimate the moments of the inverse of the determinant of the matrix $\Gamma^{i, i}$, we use standard arguments. We follow [16], Lemma 10, and the proof of (4.14) in 10 . We have the following lower bound for the determinant

$$
\begin{aligned}
\operatorname{det} \Gamma^{i, i} & \geq \inf _{\|\xi\|=1}\left(\xi^{\prime} \Gamma^{i, i} \xi\right)^{d} \\
& =\inf _{\|\xi\|=1}\left(\sum_{l=1}^{d} \int_{t_{i-1}}^{t_{i}} \int_{0}^{1}\left|\sum_{k=1}^{d} D_{r, v}^{(l)}\left(u_{k}\left(t_{i}, x\right)-u_{k}\left(t_{i-1}, x\right)\right) \xi_{k}\right|^{2} d v d r\right)^{d} .
\end{aligned}
$$

Using (2.14), (2.15), and (2.16), we get for all $x \in(0,1), t_{i-1}<r<t_{i}$,

$$
D_{r, v}^{(l)}\left(u_{k}\left(t_{i}, x\right)-u_{k}\left(t_{i-1}, x\right)\right)=G_{t_{i}-r}(x, v) \sigma_{k, l}(u(r, v))+a_{k}\left(l, r, v, t_{i}, x\right),
$$

where $a_{k}\left(l, r, v, t_{i}, x\right)$ is given by (2.15). According to (4.6) and $\mathbf{A 2}$, for any $h \in(0,1]$, we obtain that the expression in parentheses in (4.5) is bounded below by

$$
\begin{aligned}
& \sum_{l=1}^{d} \int_{t_{i}-h\left(t_{i}-t_{i-1}\right)}^{t_{i}} \int_{0}^{1}\left|\sum_{k=1}^{d} \xi_{k}\left(G_{t_{i}-r}(x, v) \sigma_{k, l}(u(r, v))+a_{k}\left(l, r, v, t_{i}, x\right)\right)\right|^{2} d v d r \\
& \geq \frac{\rho^{2}}{2} \int_{t_{i}-h\left(t_{i}-t_{i-1}\right)}^{t_{i}} d r \int_{0}^{1} d v G_{t_{i}-r}^{2}(x, v) \\
& \quad-\sum_{l=1}^{d} \int_{t_{i}-h\left(t_{i}-t_{i-1}\right)}^{t_{i}} d r \int_{0}^{1} d v\left|\sum_{k=1}^{d} \xi_{k} a_{k}\left(l, r, v, t_{i}, x\right)\right|^{2} \\
& \geq c \frac{\rho^{2}}{2} \sqrt{h\left(t_{i}-t_{i-1}\right)}-I_{h},
\end{aligned}
$$

where

$$
I_{h}=\sup _{\|\xi\|=1} \sum_{l=1}^{d} \int_{t_{i}-h\left(t_{i}-t_{i-1}\right)}^{t_{i}} d r \int_{0}^{1} d v\left|\sum_{k=1}^{d} \xi_{k} a_{k}\left(l, r, v, t_{i}, x\right)\right|^{2} .
$$

We choose $y$ such that $c \rho^{2} \sqrt{h\left(t_{i}-t_{i-1}\right)}=4 y^{-1 / d}$, and point out that since $h \leq 1$, we have $y \geq a:=4^{d} c^{-d} \rho^{-2 d}\left(t_{i}-t_{i-1}\right)^{-d / 2}$. Furthermore, as $h$ varies in $(0,1], y$ varies in $[a, \infty)$. By Chebyshev's inequality, we get that for any $q \geq 1$,

$$
\begin{aligned}
\mathbb{P}\left[\operatorname{det} \Gamma^{i, i}<\frac{1}{y}\right] & \leq \mathbb{P}\left[\left(c \frac{\rho^{2}}{2} \sqrt{h\left(t_{i}-t_{i-1}\right)}-I_{h}\right)^{d}<\frac{1}{y}\right] \\
& =\mathbb{P}\left[I_{h}>y^{-1 / d}\right] \leq y^{q / d} \mathbb{E}\left[\left|I_{h}\right|^{q}\right] .
\end{aligned}
$$

Using (2.15) and standard arguments, we find

$$
\mathbb{E}\left[\left|I_{h}\right|^{q}\right] \leq K\left(\mathbb{E}\left[\left|R_{1}\right|^{q}\right]+\mathbb{E}\left[\left|R_{2}\right|^{q}\right]\right)
$$


where

$R_{1}=\sum_{l, k, j=1}^{d} \int_{t_{i}-h\left(t_{i}-t_{i-1}\right)}^{t_{i}} d r \int_{0}^{1} d v \Lambda_{1}^{2}$ and $R_{2}=\sum_{l, k=1}^{d} \int_{t_{i}-h\left(t_{i}-t_{i-1}\right)}^{t_{i}} d r \int_{0}^{1} d v \Lambda_{2}^{2}$,

with

$$
\Lambda_{1}=\int_{r}^{t_{i}} \int_{0}^{1} G_{t_{i}-\tau}(x, z) D_{r, v}^{(l)}\left(\sigma_{k, j}(u(\tau, z))\right) W^{j}(d \tau, d z)
$$

and

$$
\Lambda_{2}=\int_{r}^{t_{i}} \int_{0}^{1} G_{t_{i}-\tau}(x, z) D_{r, v}^{(l)}\left(b_{k}(u(\tau, z))\right) d z d \tau .
$$

We bound the $q$-th moment of $R_{1}$ and $R_{2}$ separately. Concerning $R_{1}$, we utilize Burkholder's inequality for Hilbert space valued martingales [3, Eq.(4.18)] to get

$$
\begin{aligned}
& \mathbb{E}\left[\left|R_{1}\right|^{q}\right] \leq \\
& K \sum_{l, k, j=1}^{d} \mathbb{E}\left[\left|\int_{t_{i}-h\left(t_{i}-t_{i-1}\right)}^{t_{i}} d \tau \int_{0}^{1} d z G_{t_{i}-\tau}^{2}(x, z) \int_{t_{i}-h\left(t_{i}-t_{i-1}\right)}^{\tau} d r \int_{0}^{1} d v \Theta^{2}\right|^{q}\right],
\end{aligned}
$$

where

$$
\begin{aligned}
\Theta:=\left|D_{r, v}^{(l)} \sigma_{k, j}(u(\tau, z))\right| & =\left|\sum_{m=1}^{d} \frac{\partial \sigma_{k, j}}{\partial x_{m}}(u(\tau, z)) D_{r, v}^{(l)} u_{m}(\tau, z)\right| \\
& \leq K \sum_{m=1}^{d}\left|D_{r, v}^{(l)} u_{m}(\tau, z)\right|,
\end{aligned}
$$

thanks to the hypothesis A1. Therefore,

$$
\begin{aligned}
& \mathbb{E}\left[\left|R_{1}\right|^{q}\right] \leq \\
& K \sum_{l=1}^{d} \mathbb{E}\left[\left|\int_{t_{i}-h\left(t_{i}-t_{i-1}\right)}^{t_{i}} d \tau \int_{0}^{1} d z G_{t_{i}-\tau}^{2}(x, z) \int_{t_{i}-h\left(t_{i}-t_{i-1}\right)}^{\tau} d r \int_{0}^{1} d v \Psi^{2}\right|^{q}\right],
\end{aligned}
$$

where $\Psi=\sum_{m=1}^{d}\left|D_{r, v}^{(l)} u_{m}(\tau, z)\right|$. Now we use Hölder's inequality w.r.t. the measure $G_{t_{i}-\tau}^{2}(x, z) d \tau d z$ to obtain that

$$
\begin{aligned}
\mathbb{E}\left[\left|R_{1}\right|^{q}\right] & \leq K\left|\int_{t_{i}-h\left(t_{i}-t_{i-1}\right)}^{t_{i}} d \tau \int_{0}^{1} d z G_{t_{i}-\tau}^{2}(x, z)\right|^{q-1} \\
& \cdot \int_{t_{i}-h\left(t_{i}-t_{i-1}\right)}^{t_{i}} d \tau \int_{0}^{1} d z G_{t_{i}-\tau}^{2}(x, z) \sum_{l=1}^{d} \mathbb{E}\left[\left|\int_{t_{i}-h\left(t_{i}-t_{i-1}\right)}^{t_{i}} d r \int_{0}^{1} d v \Psi^{2}\right|^{q}\right] .
\end{aligned}
$$


According to Lemma 2.10 and the properties of $G$, we have

$$
\begin{aligned}
\mathbb{E}\left[\left|R_{1}\right|^{q}\right] & \leq K\left(h\left(t_{i}-t_{i-1}\right)\right)^{\frac{q-1}{2}}\left(h\left(t_{i}-t_{i-1}\right)\right)^{q / 2} \int_{t_{i}-h\left(t_{i}-t_{i-1}\right)}^{t_{i}} d \tau \int_{0}^{1} d z G_{t_{i}-\tau}^{2}(x, z) \\
& \leq K\left(h\left(t_{i}-t_{i-1}\right)\right)^{q} .
\end{aligned}
$$

As regards $R_{2}$, we derive a similar bound. By the Cauchy-Schwarz inequality,

$\mathbb{E}\left[\left|R_{2}\right|^{q}\right] \leq K\left(h\left(t_{i}-t_{i-1}\right)\right)^{q} \sum_{l, k=1}^{d} \mathbb{E}\left[\left|\int_{t_{i}-h\left(t_{i}-t_{i-1}\right)}^{t_{i}} d r \int_{0}^{1} d v \int_{r}^{t_{i}} d \tau \int_{0}^{1} d z \Phi^{2}\right|^{q}\right]$,

where $\Phi=G_{t_{i}-\tau}(x, z)\left|D_{r, v}^{(l)}\left(b_{k}(u(\tau, z))\right)\right|$. From now on, the $q$-th moment of $R_{2}$ is estimated as that of $R_{1}$ (see (4.8)), and this yields

$$
\mathbb{E}\left[\left|R_{2}\right|^{q}\right] \leq K\left(h\left(t_{i}-t_{i-1}\right)\right)^{2 q} .
$$

Hence, we have shown that

$$
\mathbb{E}\left[\left|I_{h}\right|^{q}\right] \leq K\left(h\left(t_{i}-t_{i-1}\right)\right)^{q}=K \frac{4^{2 q}}{c^{2 q} \rho^{4 q}} y^{-2 q / d} .
$$

Consequently, taking $q>\tilde{p} d$,

$$
\begin{aligned}
& \mathbb{E}\left[\left|\left(\operatorname{det} \Gamma^{i, i}\right)^{-1}\right|^{\tilde{p}}\right] \\
& =\int_{0}^{\infty} \tilde{p} y^{\tilde{p}-1} \mathbb{P}\left[\left(\operatorname{det} \Gamma^{i, i}\right)^{-1}>y\right] d y \\
& \leq a^{\tilde{p}}+\tilde{p} \int_{a}^{\infty} y^{\tilde{p}-1} \mathbb{P}\left[\operatorname{det} \Gamma^{i, i}<\frac{1}{y}\right] d y \\
& \leq \frac{4^{\tilde{p} d}}{c^{\tilde{p} d} \rho^{2 \tilde{p} d}\left(t_{i}-t_{i-1}\right)^{\tilde{p} d / 2}}+\tilde{p} \int_{a}^{\infty} y^{\tilde{p}-1+(q / d)} \mathbb{E}\left[\left|I_{h}\right|^{q}\right] d y \\
& \leq \frac{4^{\tilde{p} d}}{c^{\tilde{p} d} \rho^{2 \tilde{p} d}\left(t_{i}-t_{i-1}\right)^{\tilde{p} d / 2}}+\tilde{p} K \frac{4^{2 q}}{c^{2 q} \rho^{4 q}} \int_{a}^{\infty} y^{\tilde{p}-1+(q / d)-2(q / d)} d y \\
& \leq K^{\prime}\left(t_{i}-t_{i-1}\right)^{-\tilde{p} d / 2},
\end{aligned}
$$

where $K^{\prime}$ is a finite positive constant. Thus, we have proved that

$$
\mathbb{E}\left[\left|\left(\operatorname{det} \Gamma^{i, i}\right)^{-1}\right|^{\tilde{p}}\right] \leq K^{\prime}\left(t_{i}-t_{i-1}\right)^{-\tilde{p} d / 2} .
$$

Now, turning to the second term in (4.4), we claim that for any $l=1, \cdots, k$

$$
\mathbb{E}\left[\left\|D^{l}\left(\operatorname{det} \Gamma^{i, i}\right)^{-1}\right\|_{\mathcal{H} \otimes l}^{\tilde{p}}\right] \leq K\left(t_{i}-t_{i-1}\right)^{-\tilde{p} d / 2},
$$

for some positive finite constant $K$. Indeed, by iterating the equality (see 19 , Lemma 2.1.6])

$$
D\left(\left(\operatorname{det} \Gamma^{i, i}\right)^{-1}\right)=-\left(\operatorname{det} \Gamma^{i, i}\right)^{-2} D\left(\operatorname{det} \Gamma^{i, i}\right),
$$


we get

$$
\begin{aligned}
& D^{l}\left(\left(\operatorname{det} \Gamma^{i, i}\right)^{-1}\right) \\
& =\sum_{r=1}^{l} \sum_{\substack{l_{1}+, \ldots+l_{r}=l \\
l_{k} \geq 1, k=1, \cdots, r}} c_{r, l_{1}, \cdots, l_{r}}\left(\operatorname{det} \Gamma^{i, i}\right)^{-(r+1)} D^{l_{1}}\left(\operatorname{det} \Gamma^{i, i}\right) \otimes \cdots \otimes D^{l_{r}}\left(\operatorname{det} \Gamma^{i, i}\right) .
\end{aligned}
$$

Using Hölder's inequality, we obtain

$$
\begin{aligned}
& \mathbb{E}\left[\left\|D^{l}\left(\operatorname{det} \Gamma^{i, i}\right)^{-1}\right\|_{\mathcal{H} \otimes l}^{\tilde{p}}\right] \leq K \sum_{r=1}^{l} \sum_{\substack{l_{1}+\ldots, l_{r}=l \\
l_{k} \geq 1, k=1, \cdots, r}} \mathbb{E}\left[\left|\left(\operatorname{det} \Gamma^{i, i}\right)^{-1}\right|^{\tilde{p}(r+1)^{2}}\right]^{1 /(r+1)} \\
& \times \mathbb{E}\left[\left\|D^{l_{1}}\left(\operatorname{det} \Gamma^{i, i}\right)\right\|_{\mathcal{H} \otimes l_{1}}^{\tilde{p}(r+1)}\right]^{1 /(r+1)} \times \cdots \times \mathbb{E}\left[\left\|D^{l_{r}}\left(\operatorname{det} \Gamma^{i, i}\right)\right\|_{\mathcal{H}}^{\tilde{p}(r+1)}\right]^{1 /(r+1)} .
\end{aligned}
$$

According to (4.10), we have

$$
\mathbb{E}\left[\left|\left(\operatorname{det} \Gamma^{i, i}\right)^{-1}\right|^{\tilde{p}(r+1)^{2}}\right]^{1 /(r+1)} \leq K\left(t_{i}-t_{i-1}\right)^{-\tilde{p}(r+1) d / 2},
$$

for some constant $K>0$.

For the other factors, we write

$$
\operatorname{det} \Gamma^{i, i}=\sum_{\eta \in \Pi} \varepsilon(\eta) \prod_{k=1}^{d} \Gamma_{k, \eta(k)}^{i, i},
$$

where $\Pi=\{\eta ; \eta$ permutation of $\{1, \cdots, d\}\}$. Therefore

$$
\begin{array}{r}
\mathbb{E}\left[\left\|D^{l}\left(\operatorname{det} \Gamma^{i, i}\right)\right\|_{\mathcal{H}^{\otimes l}}^{\tilde{p}}\right] \\
\leq K \sum_{\eta \in \Pi} \sum_{\substack{l_{1}+\ldots+l_{d}=l \\
l_{r} \geq 0, r=1, \cdots, d}} \mathbb{E}\left[\left\|D^{l_{1}}\left(\Gamma_{1, \eta(1)}^{i, i}\right)\right\|_{\mathcal{H}^{\otimes l_{1}}}^{d \tilde{p}}\right]^{1 / d} \times \cdots \\
\times \mathbb{E}\left[\left\|D^{l_{d}}\left(\Gamma_{d, \eta(d)}^{i, i}\right)\right\|_{\mathcal{H}^{\otimes l_{d}}}^{d \tilde{p}}\right]^{1 / d} .
\end{array}
$$

Combining (4.2), Lemma 3.5, and Proposition 4.3, we get

$$
\mathbb{E}\left[\left\|D^{l}\left(\operatorname{det} \Gamma^{i, i}\right)\right\|_{\mathcal{H} \otimes l}^{\tilde{p}}\right] \leq K\left(t_{i}-t_{i-1}\right)^{\tilde{p} d / 2} .
$$

Then

$$
\mathbb{E}\left[\left\|D^{l}\left(\operatorname{det} \Gamma^{i, i}\right)\right\|_{\mathcal{H}^{\otimes l}}^{\tilde{p}(r+1)}\right]^{1 /(r+1)} \leq K\left(t_{i}-t_{i-1}\right)^{\tilde{p} d / 2},
$$

where $K$ is a positive constant.

By (4.12), (4.13), and (4.14), we obtain (4.11). Finally, substituting (4.10) and (4.11) into (4.4), we conclude the proof of Theorem 4.5. 
Lemma 4.6 Assume A1 and A2. Let $\pi_{n}=\left(t_{1},, \cdots, t_{n}\right)$ with $0=t_{0}<$ $t_{1}<\cdots<t_{n} \leq T, x \in(0,1), Z$ given by (4.1), and $\beta=\left(\beta_{1}, \cdots, \beta_{m}\right)$ with $\beta_{\theta}=\left(i_{\theta}, k_{\theta}\right) \in\{1, \cdots, n\} \times\{1, \cdots, d\}$, for $\theta=1, \cdots, m$, then there exists a constant $C>0$ such that

$$
\left\|H_{\pi_{n}}^{\beta}(Z, 1)\right\|_{0,2} \leq C \prod_{\theta=1}^{m}\left(t_{i_{\theta}}-t_{i_{\theta}-1}\right)^{-1 / 4} .
$$

Proof By Lemma 3.6 we have

$$
\begin{aligned}
\left\|H_{\pi_{n}}^{\beta}(Z, 1)\right\|_{0,2} \leq & C\left\|\left(\operatorname{det} \Gamma_{Z, \pi_{n}}\right)^{-1}\right\|_{m, 2^{m+2}}^{m} \\
& \times \prod_{\theta=1}^{m}\left\|D\left(u_{k_{\theta}}\left(t_{i_{\theta}}, x\right)-u_{k_{\theta}}\left(t_{i_{\theta}-1}, x\right)\right)\right\|_{m, 2^{2(m+n d)}, \mathcal{H}} \\
& \times \prod_{\left(i_{0}, k_{0}\right) \in O_{\left(i_{\theta}, k_{\theta}\right)}}\left\|D\left(u_{k_{0}}\left(t_{i_{0}}, x\right)-u_{k_{0}}\left(t_{i_{0}-1}, x\right)\right)\right\|_{m, 2^{2(m+n d)}, \mathcal{H}}^{2},
\end{aligned}
$$

where $O_{\left(i_{\theta}, k_{\theta}\right)}$ is given in Lemma 3.6. Hence, according to Proposition 4.3 and Theorem 4.5. we get

$$
\begin{aligned}
&\left\|H_{\pi_{n}}^{\beta}(Z, 1)\right\|_{0,2} \leq \tilde{C} \prod_{j=1}^{n}\left(t_{j}-t_{j-1}\right)^{-m d / 2} \prod_{\theta=1}^{m}\left(t_{i_{\theta}}-t_{i_{\theta}-1}\right)^{1 / 4} \times \prod_{\left(i_{0}, k_{0}\right) \in O_{\left(i_{\theta}, k_{\theta}\right)}}\left(t_{i_{0}}-t_{i_{0}-1}\right)^{1 / 2} \\
&= \tilde{C} \prod_{j=1}^{n}\left(t_{j}-t_{j-1}\right)^{-m d / 2} \prod_{\theta=1}^{m}\left(t_{i_{\theta}}-t_{i_{\theta}-1}\right)^{-1 / 4}\left(t_{i_{\theta}}-t_{i_{\theta}-1}\right)^{1 / 2} \\
& \times \tilde{C} \prod_{j=1}^{n}\left(t_{j}-t_{j-1}\right)^{-m d / 2} \prod_{\theta=1}^{m}\left(t_{i_{\theta}}-t_{i_{\theta}-1}\right)^{-1 / 4} \\
& \times \prod_{\left(i_{0}, k_{0}\right) \in O_{\left(i_{\theta}, k_{\theta}\right)}}^{m}\left(t_{i_{0}}-t_{i_{0}-1}\right)^{1 / 2} \\
&\left.\quad t_{i_{\theta}-1}\right)^{1 / 2} \prod_{\left(i_{0}, k_{0}\right) \in O_{\left(i_{\theta}, k_{\theta}\right)}}\left(t_{i_{0}}-t_{i_{0}-1}\right)^{1 / 2} .
\end{aligned}
$$

We remark that $\left(t_{i_{\theta}}-t_{i_{\theta}-1}\right)^{1 / 2} \prod_{\left(i_{0}, k_{0}\right) \in O_{\left(i_{\theta}, k_{\theta}\right)}}\left(t_{i_{0}}-t_{i_{0}-1}\right)^{1 / 2}$ is equal to $\prod_{j=1}^{n} \prod_{k=1}^{d}\left(t_{j}-t_{j-1}\right)^{1 / 2}=\prod_{j=1}^{n}\left(t_{j}-t_{j-1}\right)^{d / 2}$. Therefore,

$$
\prod_{\theta=1}^{m}\left(t_{i_{\theta}}-t_{i_{\theta}-1}\right)^{1 / 2} \prod_{\left(i_{0}, k_{0}\right) \in O_{\left(i_{\theta}, k_{\theta}\right)}}\left(t_{i_{0}}-t_{i_{0}-1}\right)^{1 / 2}=\prod_{j=1}^{n}\left(t_{j}-t_{j-1}\right)^{m d / 2} .
$$

Hence, by combining (4.17) and (4.18), we conclude the proof of Lemma 4.6. 
Proof (Proof of Theorem 1.3(b)) By Theorem 3.7, Lemma 4.6, and [9, Ineq. (6.2)], we conclude the proof.

\section{Proof of Theorem 1.1}

In this section, we will investigate the existence of the local time and its joint continuous version for the process $\{u(t, x), t \in[0, T]\}$.

5.1 Existence of local time when $d \leq 3$

Let $\alpha \geq 0$, we define the Sobolev space $H^{\alpha}\left(\mathbb{R}^{d}\right)$ as:

$$
H^{\alpha}\left(\mathbb{R}^{d}\right)=\left\{g \in L^{2}\left(\mathbb{R}^{d}\right) ;\left(1+\|\xi\|^{2}\right)^{\frac{\alpha}{2}} \hat{g} \in L^{2}\left(\mathbb{R}^{d}\right)\right\},
$$

where $\|\cdot\|$ is the Euclidean norm on $\mathbb{R}^{d}$ and $\hat{g}$ is the Fourier transform of $g$.

Now we give our result concerning the existence of local time of the solution to Eq. (1.1).

Theorem 5.1 Let $u(t, x)$ be given by (2.10). Assume that $d \leq 3$, then for each $x \in(0,1)$, the process $\{u(t, x), t \in[0, T]\}$ has a local time $L(\xi, t)$. Moreover, for every fixed $t, L(\bullet, t) \in H^{\alpha}\left(\mathbb{R}^{d}\right)$ for $\alpha<\frac{4-d}{2}$.

Proof Let $t \in[0, T]$, and define $f$ by

$$
f(\xi)=\int_{0}^{t} e^{i\langle\xi, u(s, x)\rangle} d s .
$$

Note that $f$ coincides with the Fourier transform of the local time,$L(\bullet, t)$, whenever $L(\bullet, t)$ exists. Since $f$ is a continuous function, then we have just to look for $\alpha$ such that

$$
\int_{\mathbb{R}^{d}}\|\xi\|^{2 \alpha}|f(\xi)|^{2} d \xi<\infty
$$

We have by Fubini's theorem

$$
\begin{aligned}
\mathbb{E}\left[\int_{\mathbb{R}^{d}}\|\xi\|^{2 \alpha}|f(\xi)|^{2} d \xi\right] & =\int_{\mathbb{R}^{d}}\|\xi\|^{2 \alpha} \int_{[0, t]^{2}} \mathbb{E}\left[e^{i\langle\xi, u(s, x)-u(r, x)\rangle}\right] d r d s d \xi \\
& \leq \int_{[0, t]^{2}} \int_{\mathbb{R}^{d}}\|\xi\|^{2 \alpha}\left|\mathbb{E}\left[e^{i\langle\xi, u(s, x)-u(r, x)\rangle}\right]\right| d \xi d r d s \\
& =2 \int_{\{0 \leq r<s \leq t\}} \int_{\mathbb{R}^{d}}\|\xi\|^{2 \alpha}\left|\mathbb{E}\left[e^{i\langle\xi, u(s, x)-u(r, x)\rangle}\right]\right| d \xi d r d s .
\end{aligned}
$$

Put $I_{1}=\left[-1 /(s-r)^{1 / 4}, 1 /(s-r)^{1 / 4}\right]$ and $I_{2}=\mathbb{R} \backslash I_{1}$, Therefore

$$
\mathbb{R}^{d}=\bigcup_{i_{1}, \cdots, i_{d} \in\{1,2\}} I_{i_{1}} \times \cdots \times I_{i_{d}} .
$$


Hence

$$
\begin{aligned}
& \mathbb{E}\left[\int_{\mathbb{R}^{d}}\|\xi\|^{2 \alpha}|f(\xi)|^{2} d \xi\right] \\
& \leq 2 \sum_{i_{1}, \cdots, i_{d} \in\{1,2\}} \int_{\{0 \leq r<s \leq t\}} \int_{I_{i_{1}} \times \cdots \times I_{i_{d}}}\|\xi\|^{2 \alpha}\left|\mathbb{E}\left[e^{i\langle\xi, u(s, x)-u(r, x)\rangle}\right]\right| d \xi d r d s .
\end{aligned}
$$

Let $\varphi_{\xi}: \mathbb{R}^{d} \ni z \mapsto e^{i\langle\xi, z\rangle}, k=\left(k_{1}, \ldots, k_{d}\right)$, and $\partial_{z}^{k}=\prod_{l=1}^{d}\left(\frac{\partial}{\partial z_{l}}\right)^{k_{l}}$. By simple calculation, we have

$$
\mathbb{E}\left[\left(\partial_{z}^{k} \varphi_{\xi}\right)(u(s, x)-u(r, x))\right]=\left(i \xi_{1}\right)^{k_{1}} \cdots\left(i \xi_{d}\right)^{k_{d}} \mathbb{E}\left[e^{i\langle\xi, u(s, x)-u(r, x)\rangle}\right]
$$

On the other hand, by integration by parts (i.e., Proposition 3.4 ), we get

$$
\begin{aligned}
\mathbb{E}\left[\left(\partial_{z}^{k} \varphi_{\xi}\right)(u(s, x)-u(r, x))\right] & =\mathbb{E}\left[\left(\partial_{y_{2}}^{k} g\right)(u(r, x), u(s, x)-u(r, x))\right] \\
& =\mathbb{E}\left[e^{i\langle\xi, u(s, x)-u(r, x)\rangle} H_{\pi_{2}}^{\beta}(F, 1)\right],
\end{aligned}
$$

where $\pi_{2}=(r, s), F=(u(r, x), u(s, x)-u(r, x)), g: \mathbb{R}^{d} \times \mathbb{R}^{d} \ni\left(y_{1}, y_{2}\right) \mapsto$ $\varphi_{\xi}\left(y_{2}\right), y_{i}=\left(y_{i, 1}, \ldots, y_{i, d}\right)$, for $i=1,2, \partial_{y_{2}}^{k}=\prod_{l=1}^{d}\left(\frac{\partial}{\partial y_{2, l}}\right)^{k_{l}}, m=\sum_{l=1}^{d} k_{l}$, and

$$
\beta=(\underbrace{(2,1), \cdots,(2,1)}_{k_{1} \text { times }}, \cdots, \underbrace{(2, d), \cdots,(2, d)}_{k_{d} \text { times }}) .
$$

Combining (5.2) and (5.3), we obtain

$$
\begin{aligned}
\left|\mathbb{E}\left[e^{i\langle\xi, u(s, x)-u(r, x)\rangle}\right]\right| & =\left|\xi_{1}\right|^{-k_{1}} \cdots\left|\xi_{d}\right|^{-k_{d}}\left|\mathbb{E}\left[e^{i\langle\xi, u(s, x)-u(r, x)\rangle} H_{\pi_{2}}^{\beta}(F, 1)\right]\right| \\
& \leq\left|\xi_{1}\right|^{-k_{1}} \ldots\left|\xi_{d}\right|^{-k_{d}} \mathbb{E}\left[\left|H_{\pi_{2}}^{\beta}(F, 1)\right|\right] .
\end{aligned}
$$

According to 4.15), we write

$$
\left\|H_{\pi_{2}}^{\beta}(F, 1)\right\|_{0,2} \leq C \prod_{\theta=1}^{m}(s-r)^{-1 / 4}=C(s-r)^{-\sum_{l=1}^{d} k_{l} / 4} .
$$

Therefore, according to (5.4) and (5.5), we get for all positive integers $k_{1}, \cdots, k_{d}$, there exists a positive constant $C=C\left(k_{1}, \cdots, k_{d}\right)$, such that

$$
\left|\mathbb{E}\left[e^{i\langle\xi, u(s, x)-u(r, x)\rangle}\right]\right| \leq \frac{C}{\left|\xi_{1}\right|^{k_{1}} \cdots\left|\xi_{d}\right|^{k_{d}}(s-r)^{\sum_{l=1}^{d} k_{l} / 4}},
$$

here $\xi=\left(\xi_{1}, \cdots, \xi_{d}\right)$. Put, for $l=1, \cdots, d$,

$$
k_{l}\left(i_{l}\right)= \begin{cases}0, & \text { if } \quad i_{l}=1 \\ 2([\alpha]+2), & \text { if } \quad i_{l}=2,\end{cases}
$$


where $[\alpha]$ is the integer part of $\alpha$. According to (5.1) and (5.6), we get

$$
\begin{aligned}
& \mathbb{E}\left[\int_{\mathbb{R}^{d}}\|\xi\|^{2 \alpha}|f(\xi)|^{2} d \xi\right] \\
& \leq K_{1} \sum_{i_{1}, \cdots, i_{d} \in\{1,2\}} \int_{\{0 \leq r<s \leq t\}} \int_{I_{i_{1}} \times \cdots \times I_{i_{d}}} \frac{\|\xi\|^{2 \alpha}}{\left|\xi_{1}\right|^{k_{1}\left(i_{1}\right)} \cdots\left|\xi_{d}\right|^{k_{d}\left(i_{d}\right)}(s-r)^{\sum_{l=1}^{d} k_{l}\left(i_{l}\right) / 4}} d \xi d r d s \\
& \leq K_{2} \sum_{p=1}^{d} \sum_{i_{1}, \cdots, i_{d} \in\{1,2\}} \int_{\{0 \leq r<s \leq t\}} \int_{I_{i_{1}} \times \cdots \times I_{i_{d}}} \frac{\left|\xi_{p}\right|^{2 \alpha}}{\left|\xi_{1}\right|^{k_{1}\left(i_{1}\right)} \ldots\left|\xi_{d}\right|^{k_{d}\left(i_{d}\right)}(s-r)^{\sum_{l=1}^{d} k_{l}\left(i_{l}\right) / 4}} d \xi d r d s \\
& =K_{2} \sum_{p=1}^{d} \sum_{i_{i_{1}}, \cdots, i_{d} \in\{1,2\}} \int_{\{0 \leq r<s \leq t\}} \prod_{l=1}^{p-1} \int_{I_{i_{l}}} \frac{1}{\left|\xi_{l}\right|^{k_{l}\left(i_{l}\right)}(s-r)^{k_{l}\left(i_{l}\right) / 4}} d \xi_{l} \\
& \quad \times \int_{I_{i_{p}}} \frac{\left|\xi_{p}\right|^{2 \alpha}}{\left|\xi_{p}\right|^{k_{p}\left(i_{p}\right)}(s-r)^{k_{p}\left(i_{p}\right) / 4}} d \xi_{p} \prod_{l=p+1}^{d} \int_{I_{i_{l}}} \frac{1}{\left|\xi_{l}\right|^{k_{l}\left(i_{l}\right)(s-r)^{k_{l}\left(i_{l}\right) / 4}} d \xi_{l} d r d s .}
\end{aligned}
$$

By simple calculation, we obtain

- When $i_{l}=1$ or 2 with $l \neq p$, we have $\int_{I_{i_{l}}} \frac{1}{\left|\xi_{l}\right|^{k_{l}\left(i_{l}\right)}(s-r)^{k_{l}\left(i_{l}\right) / 4}} d \xi_{l}=\frac{c}{(s-r)^{1 / 4}}$, where $c$ is a positive constant depending on $i_{l}$ and $\alpha$;

- If $i_{p}=1$ or 2, we get $\int_{I_{i_{p}}} \frac{\left|\xi_{p}\right|^{2 \alpha}}{\left|\xi_{p}\right|^{k_{p}\left(i_{p}\right)}(s-r)^{k_{p}\left(i_{p}\right) / 4}} d \xi_{p}=\frac{c^{\prime}}{(s-r)^{(2 \alpha+1) / 4}}$, where $c^{\prime}$ is a positive constant depending on $i_{p}$ and $\alpha$.

Combining the above discussion with (5.7), we have

$$
\mathbb{E}\left[\int_{\mathbb{R}^{d}}\|\xi\|^{2 \alpha}|f(\xi)|^{2} d \xi\right] \leq K_{3} \int_{\{0 \leq r<s \leq t\}} \frac{1}{(s-r)^{(2 \alpha+d) / 4}} d r d s .
$$

Hence, the local time $L(\xi, t)$ exists for $d=1,2,3$ and $L(\bullet, t) \in H^{\alpha}\left(\mathbb{R}^{d}\right)$ for $\alpha<\frac{4-d}{2}$. Which finishes the proof of Theorem [5.1]

5.2 The local time does not exist for $d \geq 4$

The below theorem is a classical result on the existence of local time for a stochastic process $X$ with values in $\mathbb{R}^{d}$.

Theorem 5.2 (Theorem 21.15 in [12]) The local time, $L(\bullet, t)$, exists with $L(\bullet, t) \in L^{2}\left(\mathbb{P} \otimes \lambda_{d}\right)$ iff

$$
\liminf _{\varepsilon \rightarrow 0} \varepsilon^{-d} \int_{0}^{t} \int_{0}^{t} \mathbb{P}\left[\left\|X_{s}-X_{r}\right\| \leq \varepsilon\right] d r d s<\infty .
$$


Taking into account the above theorem, we are ready to give the following result.

Theorem 5.3 Let $u(t, x)$ be given by (2.10). Assume that $d \geq 4$, then for each $x \in(0,1)$, the process $\{u(t, x), t \in[0, T]\}$ does not have a local time $L(\xi, t)$ in $L^{2}\left(\mathbb{P} \otimes \lambda_{d}\right)$ for any $t \in[0, T]$.

Proof We have by (1.2) and Fubini's theorem

$$
\begin{aligned}
& \int_{0}^{t} \int_{0}^{t} \mathbb{P}[\|u(s, x)-u(r, x)\| \leq \varepsilon] d r d s \\
& \geq c \int_{B(0, \varepsilon)} \int_{0}^{t} \int_{0}^{t} \frac{1}{|s-r|^{d / 4}} \exp \left(-\frac{\|y\|^{2}}{c|s-r|^{1 / 2}}\right) d r d s d y .
\end{aligned}
$$

We now fix $s$ and use the change of variables $\tau=s-r$ to see that this above expression equal to

$$
c \int_{B(0, \varepsilon)} \int_{0}^{t} \int_{-t+s}^{s} \frac{\exp \left(-\frac{\|y\|^{2}}{c|\tau|^{1 / 2}}\right)}{|\tau|^{d / 4}} d \tau d s d y
$$

Let $0<\alpha<t$, hence this above term is greater than or equal to

$$
\begin{aligned}
& c \int_{B(0, \varepsilon)} \int_{t-\alpha}^{t} \int_{0}^{s} \frac{\exp \left(-\frac{\|y\|^{2}}{c \tau^{1 / 2}}\right)}{\tau^{d / 4}} d \tau d s d y \\
& \geq c \int_{B(0, \varepsilon)} \int_{t-\alpha}^{t} \int_{0}^{t-\alpha} \frac{\exp \left(-\frac{\|y\|^{2}}{c \tau^{1 / 2}}\right)}{\tau^{d / 4}} d \tau d s d y \\
& =c_{1} \int_{B(0, \varepsilon)} \int_{0}^{t-\alpha} \frac{\exp \left(-\frac{\|y\|^{2}}{c \tau^{1 / 2}}\right)}{\tau^{d / 4}} d \tau d y .
\end{aligned}
$$

By the change of variables $\tau=c^{-2}\|y\|^{4} u$ we see that this is greater than or equal to

$$
c_{2} \int_{B(0, \varepsilon)} \frac{1}{\|y\|^{d-4}} \int_{0}^{\frac{c^{2}(t-\alpha)}{\|y\|^{4}}} \frac{\exp \left(-\frac{1}{u^{1 / 2}}\right)}{u^{d / 4}} d u d y .
$$

- If $d \geq 5$. Assume that $\varepsilon \in(0,1)$ and let $0<\beta<c^{2}(t-\alpha)$. Then (5.9) is greater than or equal to

$$
c_{2} \int_{B(0, \varepsilon)} \frac{1}{\|y\|^{d-4}} \int_{\beta}^{c^{2}(t-\alpha)} \frac{\exp \left(-\frac{1}{u^{1 / 2}}\right)}{u^{d / 4}} d u d y=c_{3} \int_{B(0, \varepsilon)} \frac{1}{\|y\|^{d-4}} d y \geq c_{3} \varepsilon^{4} .
$$


Therefore, we conclude that

$$
\liminf _{\varepsilon \rightarrow 0} \varepsilon^{-d} \int_{0}^{t} \int_{0}^{t} \mathbb{P}\left[\left\|X_{s}-X_{r}\right\| \leq \varepsilon\right] d r d s=\infty .
$$

Hence, by Theorem 5.2 the local time does not exist for $d \geq 5$.

- If $d=4$. Assume that $\varepsilon \in(0,1)$ and let $0<\beta<c^{2}(t-\alpha)$. Then (5.9) is greater than or equal to

$$
\begin{aligned}
c_{2} \int_{B(0, \varepsilon)} \int_{\beta}^{\frac{c^{2}(t-\alpha)}{\|y\|^{4}}} \frac{\exp \left(-\frac{1}{u^{1 / 2}}\right)}{u} d u d y & \geq c_{2} e^{-\frac{1}{\beta^{1 / 2}}} \int_{B(0, \varepsilon)} \log \left(\frac{c^{2}(t-\alpha)}{\beta\|y\|^{4}}\right) d y \\
& \geq c_{2} e^{-\frac{1}{\beta^{1 / 2}}} \varepsilon^{4} \log \left(\frac{c^{2}(t-\alpha)}{\beta \varepsilon^{4}}\right) .
\end{aligned}
$$

Therefore

$$
\liminf _{\varepsilon \rightarrow 0} \varepsilon^{-4} \int_{0}^{t} \int_{0}^{t} \mathbb{P}\left[\left\|X_{s}-X_{r}\right\| \leq \varepsilon\right] d r d s=\infty .
$$

Then, by Theorem 5.2 the local time does not exist for $d=4$. This concludes the proof of Theorem 5.3

\subsection{Regularity of local time}

Our goal in this section is to look for a version of the local time $L(\xi, t)$ with jointly Hölder continuity in $(\xi, t)$. Moreover, we show that the local time satisfies a Hölder condition with respect to the time variable $t$, uniformly in the space variable $\xi$. We start by proving the $\alpha$-LND property for the process $\{u(t, x), t \in[0, T]\}$.

Theorem 5.4 Let $u(t, x)$ be given by (2.10). Hence, for each fixed $x \in(0,1)$, the process $\{u(t, x), t \in[0, T]\}$ verifies the $\frac{1}{4}-L N D$ property on $[0, T]$, i.e., for every nonnegative integers $m \geq 2, k_{j, l}$, for $j=1, \cdots, m$ and $l=1, \cdots, d$, there exists a constant $c=c\left(m, k_{j, l}\right)$ such that

$$
\left|\mathbb{E}\left[e^{i \sum_{j=1}^{m}\left\langle v_{j}, u\left(t_{j}, x\right)-u\left(t_{j-1}, x\right)\right\rangle}\right]\right| \leq \frac{c}{\prod_{j=1}^{m} \prod_{l=1}^{d}\left|v_{j, l}\right|^{k_{j, l}}\left(t_{j}-t_{j-1}\right)^{k_{j, l} / 4}},
$$

for all $v_{j}=\left(v_{j, l} ; 1 \leq l \leq d\right) \in(\mathbb{R} \backslash\{0\})^{d}$, for $j=1, \cdots, m$, and for every ordered points $0=t_{0}<t_{1}<\cdots<t_{m} \leq T$.

Proof The proof is a simple consequence of the integration by parts (3.1) and (4.15).

In order to use Kolmogorov's theorem to conclude various continuities of the local time $L(\xi, t)$ in $t$ and $\xi$, we seek to estimate the moments of the increments of $L(\xi, t)$. 
Lemma 5.5 Let $u(t, x)$ be given by (2.10). Assume $d \leq 3$. Let $\tilde{L}(\xi, t)$ be given as in (2.2), therefore, for every $\xi, y \in \mathbb{R}^{d}, t, t+h \in[0, T]$, and even integer $m \geq 2$,

$$
\begin{gathered}
\mathbb{E}[\tilde{L}(\xi, t+h)-\tilde{L}(\xi, t)]^{m} \leq C_{m}|h|^{m\left(1-\frac{d}{4}\right)} ; \\
\mathbb{E}[\tilde{L}(\xi+y, t+h)-\tilde{L}(\xi, t+h)-\tilde{L}(\xi+y, t)+\tilde{L}(\xi, t)]^{m} \\
\leq C_{m, \theta}\|y\|^{m \theta}|h|^{m\left(1-\frac{d}{4}-\frac{\theta}{4}\right)},
\end{gathered}
$$

where $0<\theta<\left(\frac{4-d}{2}\right) \wedge 1$.

Proof We prove just the second inequality; the first one follows the same lines. We consider only $h>0$ such that $t+h \in[0, T]$, the other case follows the same way. According to (2.3), we get

$$
\begin{aligned}
& \mathbb{E}[\tilde{L}(\xi+y, t+h)-\tilde{L}(\xi, t+h)-\tilde{L}(\xi+y, t)+\tilde{L}(\xi, t)]^{m} \\
& =\frac{1}{(2 \pi)^{m d}} \int_{\left.\mathbb{R}^{d}\right)^{m}} \int_{[t, t+h]^{m}} \prod_{j=1}^{m}\left(e^{-i\left\langle v_{j}-v_{j+1}, \xi+y\right\rangle}-e^{-i\left\langle v_{j}-v_{j+1}, \xi\right\rangle}\right) \\
& \times \mathbb{E}\left[e^{i \sum_{j=1}^{m}\left\langle v_{j}, u\left(t_{j}, x\right)-u\left(t_{j-1}, x\right)\right\rangle}\right] \prod_{j=1}^{m} d t_{j} \prod_{j=1}^{m} d v_{j} .
\end{aligned}
$$

By the elementary inequality $\left|1-e^{i \rho}\right| \leq 2^{1-\theta}|\rho|^{\theta}$ for all $0<\theta<1$ and $\rho \in \mathbb{R}$, we have

$$
\begin{aligned}
& \mathbb{E}[\tilde{L}(\xi+y, t+h)-\tilde{L}(\xi, t+h)-\tilde{L}(\xi+y, t)+\tilde{L}(\xi, t)]^{m} \\
& \leq 2^{-m d-\theta+1} \pi^{-m d}\|y\|^{m \theta} \mathcal{J}(m, \theta)
\end{aligned}
$$

where

$$
\begin{aligned}
& \mathcal{J}(m, \theta) \\
& =\int_{[t, t+h]^{m}} \int_{\left(\mathbb{R}^{d}\right)^{m}} \prod_{j=1}^{m}\left\|v_{j}-v_{j+1}\right\|^{\theta}\left|\mathbb{E}\left[e^{i \sum_{j=1}^{m}\left\langle v_{j}, u\left(t_{j}, x\right)-u\left(t_{j-1}, x\right)\right\rangle}\right]\right| \prod_{j=1}^{m} d v_{j} \prod_{j=1}^{m} d t_{j} .
\end{aligned}
$$

We replace the integration over the domain $[t, t+h]^{m}$ by the integration over the subset $\Lambda=\left\{t \leq t_{1}<\cdots<t_{m} \leq t+h\right\}$, hence we obtain

$$
\begin{aligned}
& \mathcal{J}(m, \theta) \\
& =m ! \int_{\Lambda} \int_{\left(\mathbb{R}^{d}\right)^{m}} \prod_{j=1}^{m}\left\|v_{j}-v_{j+1}\right\|^{\theta}\left|\mathbb{E}\left[e^{i \sum_{j=1}^{m}\left\langle v_{j}, u\left(t_{j}, x\right)-u\left(t_{j-1}, x\right)\right\rangle}\right]\right| \prod_{j=1}^{m} d v_{j} \prod_{j=1}^{m} d t_{j},
\end{aligned}
$$

where $t_{0}=0$ and $v_{m+1}=0$. By the fact that $\|a-b\|^{\theta} \leq\|a\|^{\theta}+\|b\|^{\theta}$ for all $0<\theta<1$ and $a, b \in \mathbb{R}^{d}$, it follows that

$$
\prod_{j=1}^{m}\left\|v_{j}-v_{j+1}\right\|^{\theta} \leq \prod_{j=1}^{m}\left(\left\|v_{j}\right\|^{\theta}+\left\|v_{j+1}\right\|^{\theta}\right) .
$$


Note that the right side of this last inequality is at most equal to a finite sum of terms each of the form $\prod_{j=1}^{m}\left\|v_{j}\right\|^{\epsilon_{j} \theta}$, where $\epsilon_{j}=0,1$, or 2 and $\sum_{j=1}^{m} \epsilon_{j}=m$. Therefore

$$
\begin{aligned}
& \mathcal{J}(m, \theta) \leq m ! \sum_{\left(\epsilon_{1}, \cdots, \epsilon_{m}\right) \in\{0,1,2\}^{m}} \\
& \int_{\Lambda} \int_{\left(\mathbb{R}^{d}\right)^{m}} \prod_{j=1}^{m}\left\|v_{j}\right\|^{\epsilon_{j} \theta} \\
& \times\left|\mathbb{E}\left[e^{i \sum_{j=1}^{m}\left\langle v_{j}, u\left(t_{j}, x\right)-u\left(t_{j-1}, x\right)\right\rangle}\right]\right| \prod_{j=1}^{m} d v_{j} \prod_{j=1}^{m} d t_{j} .
\end{aligned}
$$

On the other hand, by the $\frac{1}{4}$-LND property, i.e., Theorem 5.4 we get for every nonnegative integers $m \geq 2, k_{j, l}$, for $j=1, \cdots, m$ and $l=1, \cdots, d$, there exists a constant $c=c\left(m, k_{j, l}\right)$ such that

$$
\left|\mathbb{E}\left[e^{i \sum_{j=1}^{m}\left\langle v_{j}, u\left(t_{j}, x\right)-u\left(t_{j-1}, x\right)\right\rangle}\right]\right| \leq \frac{c}{\prod_{j=1}^{m} \prod_{l=1}^{d}\left|v_{j, l}\right|^{k_{j, l}}\left(t_{j}-t_{j-1}\right)^{k_{j, l} / 4}},
$$

where $v_{j}=\left(v_{j, 1}, \cdots, v_{j, d}\right)$. Put $I_{1}^{j}=\left[-1 /\left(t_{j}-t_{j-1}\right)^{1 / 4}, 1 /\left(t_{j}-t_{j-1}\right)^{1 / 4}\right]$ and $I_{2}^{j}=\mathbb{R} \backslash I_{1}^{j}$, Therefore

$$
\left(\mathbb{R}^{d}\right)^{m}=\bigcup_{\substack{i_{j, l} \in\{1,2\} \\ j=1, \cdots, m ; l=1, \cdots, d}} \prod_{j=1}^{m} I_{i_{j, 1}}^{j} \times \cdots \times I_{i_{j, d}}^{j} .
$$

Set, for $j=1, \cdots, m$ and $l=1, \cdots, d$,

$$
k_{j, l}\left(i_{j, l}\right)= \begin{cases}0, \text { if } & i_{j, l}=1 \\ 4, \text { if } \quad i_{j, l}=2,\end{cases}
$$

Hence, by (5.15), (5.16), and (5.17), we obtain

$$
\begin{aligned}
\mathcal{J}(m, \theta) & \leq m ! c \sum_{\substack{i_{j, l} \in\{1,2\} \\
j=1, \cdots, m ; l=1, \cdots, d}} \sum_{\left(\epsilon_{1}, \cdots, \epsilon_{m}\right) \in\{0,1,2\}} \int_{\Lambda} \int_{\prod_{j=1}^{m} I_{i_{j, 1}}^{j} \times \cdots \times I_{i_{j, d}}^{j}} \\
& \times \frac{\prod_{j=1}^{m}\left\|v_{j}\right\|^{\epsilon_{j} \theta}}{\prod_{j=1}^{m} \prod_{l=1}^{d}\left|v_{j, l}\right|^{k_{j, l}\left(i_{j, l}\right)}\left(t_{j}-t_{j-1}\right)^{k_{j, l}\left(i_{j, l}\right) / 4}} \prod_{j=1}^{m} d v_{j} \prod_{j=1}^{m} d t_{j} .
\end{aligned}
$$

We remark that

$$
\prod_{j=1}^{m}\left\|v_{j}\right\|^{\epsilon_{j} \theta} \leq \prod_{j=1}^{m}\left(\left|v_{j, 1}\right|^{\epsilon_{j} \theta}+\cdots+\left|v_{j, d}\right|^{\epsilon_{j} \theta}\right)=\sum_{l_{1}, \cdots, l_{d} \in\{1, \cdots, d\}} \prod_{j=1}^{m}\left|v_{j, l_{j}}\right|^{\epsilon_{j} \theta} .
$$

Therefore

$$
\begin{aligned}
\mathcal{J}(m, \theta) & \leq m ! c \sum_{l_{1}, \cdots, l_{d} \in\{1, \cdots, d\}} \sum_{\substack{i_{j, l} \in\{1,2\} \\
j=1, \cdots, m ; l=1, \cdots, d}} \sum_{\left(\epsilon_{1}, \cdots, \epsilon_{m}\right) \in\{0,1,2\}} \int_{\Lambda} \int_{\prod_{j=1}^{m} I_{i_{j, 1}}^{j} \times \cdots \times I_{i_{j, d}}^{j}} \\
& \times \frac{\prod_{j=1}^{m}\left|v_{j, l_{j}}\right| \epsilon_{j} \theta}{\prod_{j=1}^{m} \prod_{l=1}^{d}\left|v_{j, l}\right|^{k_{j, l}\left(i_{j, l}\right)}\left(t_{j}-t_{j-1}\right)^{k_{j, l}\left(i_{j, l}\right) / 4}} \prod_{j=1}^{m} d v_{j} \prod_{j=1}^{m} d t_{j} .
\end{aligned}
$$


According to Fubini's theorem, the right side of the above expression is equal to

$$
\begin{aligned}
& m ! c \sum_{l_{1}, \cdots, l_{d} \in\{1, \cdots, d\}} \sum_{\substack{i_{j, l} \in\{1,2\} \\
j=1, \cdots, m ; l=1, \cdots, d}} \sum_{\left(\epsilon_{1}, \cdots, \epsilon_{m}\right) \in\{0,1,2\} m} \int_{\Lambda} \prod_{j=1}^{m} \int_{I_{i_{j, 1}^{j}}^{j} \times \cdots \times I_{i_{j, d}}^{j}} \\
& \times \frac{\left|v_{j, l_{j}}\right|^{\epsilon_{j} \theta}}{\prod_{l=1}^{d}\left|v_{j, l}\right|^{k_{j, l}\left(i_{j, l}\right)}\left(t_{j}-t_{j-1}\right)^{k_{j, l}\left(i_{j, l}\right) / 4}} d v_{j} \prod_{j=1}^{m} d t_{j} . \\
& =m ! c \sum_{l_{1}, \cdots, l_{d} \in\{1, \cdots, d\}} \sum_{\substack{i_{j, l} \in\{1,2\} \\
j=1, \cdots, m ; l=1, \cdots, d}} \sum_{\left(\epsilon_{1}, \cdots, \epsilon_{m}\right) \in\{0,1,2\}^{m}} \\
& \times \int_{\Lambda} \prod_{j=1}^{m} \prod_{\substack{l=1 \\
l \neq l_{j}}}^{d} \int_{I_{i_{j, l}}^{j}} \frac{1}{\left|v_{j, l}\right|^{k_{j, l}\left(i_{j, l}\right)}\left(t_{j}-t_{j-1}\right)^{k_{j, l}\left(i_{j, l}\right) / 4}} d v_{j, l} \\
& \times \int_{I_{i_{j, l_{j}}}^{j}} \frac{1}{\left|v_{j, l_{j}}\right|^{k_{j, l_{j}}\left(i_{j, l_{j}}\right)-\epsilon_{j} \theta}\left(t_{j}-t_{j-1}\right)^{k_{j, l_{j}}\left(i_{j, l_{j}}\right) / 4}} d v_{j, l_{j}} \prod_{j=1}^{m} d t_{j} .
\end{aligned}
$$

- If $i_{j, l}=1$ or 2 with $l \neq l_{j}$, then we have

$$
\int_{I_{i_{j, l}^{j}}^{j}} \frac{1}{\left|v_{j, l}\right|^{k_{j, l}\left(i_{j, l}\right)}\left(t_{j}-t_{j-1}\right)^{k_{j, l}\left(i_{j, l}\right) / 4}} d v_{j, l}=\frac{K_{1}}{\left(t_{j}-t_{j-1}\right)^{1 / 4}},
$$

where the constant $K_{1}$ depends only on $i_{j, l}$.

- If $i_{j, l_{j}}=1$ or 2 , then we get

$$
\int_{I_{i_{j, l_{j}}}^{j}} \frac{1}{\left|v_{j, l_{j}}\right|^{k_{j, l_{j}}\left(i_{j, l_{j}}\right)-\epsilon_{j} \theta}\left(t_{j}-t_{j-1}\right)^{k_{j, l_{j}}\left(i_{j, l_{j}}\right) / 4}} d v_{j, l_{j}}=\frac{K_{2}}{\left(t_{j}-t_{j-1}\right)^{\left(1+\epsilon_{j} \theta\right) / 4}},
$$

where the constant $K_{2}$ depends on $i_{j, l_{j}}, \theta$, and $\epsilon_{j}$ such that $\sup _{\theta, \epsilon_{j}} K_{2}<\infty$.

Combining the above discussion with (5.19), we obtain

$$
\begin{aligned}
\mathcal{J}(m, \theta) & \leq m ! c_{1} \sum_{l_{1}, \cdots, l_{d} \in\{1, \cdots, d\}} \sum_{\substack{i_{j, l} \in\{1,2\} \\
j=1, \cdots, m ; l=1, \cdots, d}} \sum_{\left(\epsilon_{1}, \cdots, \epsilon_{m}\right) \in\{0,1,2\}^{m}} \\
& \times \int_{\Lambda} \prod_{j=1}^{m} \frac{1}{\left(t_{j}-t_{j-1}\right)^{\left(d+\epsilon_{j} \theta\right) / 4}} \prod_{j=1}^{m} d t_{j} .
\end{aligned}
$$

According to an elementary calculation (cf. Ehm [11]), for every $m \geq 1, h>0$, and $b_{j}<1$,

$$
\int_{t \leq s_{1}<\cdots<s_{m} \leq t+h} \prod_{j=1}^{m} \frac{1}{\left(s_{j}-s_{j-1}\right)^{b_{j}}} \prod_{j=1}^{m} d s_{j}=h^{m-\sum_{j=1}^{m} b_{j}} \frac{\prod_{j=1}^{m} \Gamma\left(1-b_{j}\right)}{\Gamma\left(1+k-\sum_{j=1}^{m} b_{j}\right)},
$$


where $s_{0}=t$. By (5.20) and (5.21), it follows that for $0<\theta<\left(\frac{4-d}{2}\right) \wedge 1$ and $b_{j}=\frac{d+\epsilon_{j} \theta}{4}$,

$$
\mathcal{J}(m, \theta) \leq \tilde{C}(m, \theta) h^{m\left(1-\frac{d+\theta}{4}\right)} .
$$

Finally, by (5.13) we get

$\mathbb{E}[\tilde{L}(\xi+y, t+h)-\tilde{L}(\xi, t+h)-\tilde{L}(\xi+y, t)+\tilde{L}(\xi, t)]^{m} \leq C(m, \theta)\|y\|^{m \theta} h^{m\left(1-\frac{d+\theta}{4}\right)}$.

Which finishes the proof of Lemma 5.5 .

Proof (Proof of Theorem 1.1 (ii)) The proof is a consequence of Lemma 5.5 and [6, Theorem 3.1].

Proof (Proof of Corollary 1.2) The proof is a simple application of Theorem 2.6 and Theorem 1.1 (ii).

Remark 5.6 Let $Y=\left(Y_{t}\right)_{t \in[0, T]}$ be an $\mathbb{R}^{d}$-valued stochastic process which is $\alpha-L N D$ with $\alpha \in(0,1)$. By the same calculations as in Section [5, we can get the following results:

1. Assume that $d<\frac{1}{\alpha}$. Then the process $Y$ has a local time $L(\xi, t)$. Moreover, for every fixed $t, L(\bullet, t) \in H^{\beta}\left(\mathbb{R}^{d}\right)$ for $\beta<\left(\frac{1}{\alpha}-d\right) / 2$, here $H^{\beta}\left(\mathbb{R}^{d}\right)$ is the Sobolev space of index $\beta$.

2. Assume $d<\frac{1}{\alpha}$. The local time of the process $Y$ has a version, denoted by $L(\xi, t)$, which is $s$ jointly continuous in $(\xi, t)$ almost surely, and which is $\gamma$-Hölder continuous in $t$, uniformly in $\xi$, for all $\gamma<1-d \alpha$ : there exist two random variables $\eta$ and $\delta$ which are almost surely finite and positive such that

$$
\sup _{\xi \in \mathbb{R}^{d}}|L(\xi, t+h)-L(\xi, t)| \leq \eta|h|^{\gamma},
$$

for all $t, t+h \in[0, T]$ and all $|h|<\delta$.

Acknowledgements The authors would like to acknowledge the comments, questions, and remarks of the referees. Their help improved the quality and clarity of this paper.

\section{Availability of data and materials}

Data sharing not applicable to this article as no datasets were generated or analysed during the current study.

\section{References}

1. Adler, R.J.: The Geometry of Random Fields. Wiley, New York (1981)

2. Bally, V., Millet, A., Sanz-Sole, M.: Approximation and support theorem in Holder norm for parabolic stochastic partial differential equations. Ann. Probab. 23(1), 178$222(1995)$

3. Bally, V., Pardoux, E.: Malliavin calculus for white noise driven parabolic SPDEs. Potential Anal. 9(1), 27-64 (1998) 
4. Berman, S.M.: Harmonic analysis of local times and sample functions of Gaussian processes. Trans. Am. Math. Soc. 143, 269 (1969)

5. Berman, S.M.: Local times and sample function properties of stationary Gaussian processes. Trans. Am. Math. Soc. 137, 277 (1969)

6. Berman, S.M.: Gaussian sample functions: Uniform dimension and Hölder conditions nowhere. Nagoya Math. J. 46, 63-86 (1972)

7. Berman, S.M.: Local nondeterminism and local times of general stochastic processes. Annales de l'I.H.P. Probabilités et statistiques 19(2), 189-207 (1983)

8. Berman, S.M., Getoor, R.: Local nondeterminism and local times of gaussian processes. Indiana University Mathematics Journal 23(1), 69-94 (1973)

9. Dalang, R.C., Khoshnevisan, D., Nualart, E.: Hitting probabilities for systems of nonlinear stochastic heat equations with multiplicative noise. Probab. Theory Relat. Fields 144(3-4), 371-427 (2009)

10. Dalang, R.C., Nualart, E.: Potential theory for hyperbolic SPDEs. Ann. Probab. 32(3 A), 2099-2148 (2004)

11. Ehm, W.: Sample function properties of multi-parameter stable processes. Zeitschrift für Wahrscheinlichkeitstheorie und Verwandte Gebiete 56(2), 195-228 (1981)

12. Geman, D., Horowitz, J.: Occupation densities. Ann. Probab. 8(1), 1-67 (1980)

13. Kerchev, G., Nourdin, I., Saksman, E., Viitasaari, L.: Local times and sample path properties of the Rosenblatt process. Stoch. Process. their Appl. 131, 498-522 (2021)

14. Kohatsu-Higa, A.: Lower bounds for densities of uniformly elliptic random variables on Wiener space. Probab. Theory Relat. Fields 126(3), 421-457 (2003)

15. Lou, S., Ouyang, C.: Local times of stochastic differential equations driven by fractional Brownian motions. Stoch. Process. their Appl. 127(11), 3643-3660 (2017)

16. Moret, S., Nualart, D.: Generalization of Itô's formula for smooth nondegenerate martingales. Stoch. Process. their Appl. 91(1), 115-149 (2001)

17. Morien, P.L.: The Hölder and the Besov regularity of the density for the solution of a parabolic stochastic partial differential equation. Bernoulli 5(2), 275-298 (1999)

18. Nolan, J.P.: Local nondeterminism and local times for stable processes. Probability theory and related fields $\mathbf{8 2}(3), 387-410$ (1989)

19. Nualart, D.: The Malliavin Calculus and Related Topics. Springer (2006)

20. Sanz-Solé, M.: Malliavin Calculus: With Applications to Stochastic Partial Differential Equations. EPFL Press (2005)

21. Walsh, J.B.: An introduction to stochastic partial differential equations. In: École d'Été Probab. Saint Flour XIV - 1984, pp. 265-439 (2006)

22. Watanabe, S.: Lectures on stochastic differential equations and Malliavin calculus, vol. 164. Springer Berlin Heidelberg New York (1984) 